This is the final peer-reviewed accepted manuscript of:

Bin, M., Marconi, L. and Teel, A. R. (2019) 'Adaptive output regulation for linear systems via discrete-time identifiers', Automatica, p. 422.

The final published version is available online at: https://doi.org/10.1016/j.automatica.2019.04.019

Rights / License:

The terms and conditions for the reuse of this version of the manuscript are specified in the publishing policy. For all terms of use and more information see the publisher's website. 


\title{
Adaptive Output Regulation for Linear Systems via Discrete-Time Identifiers
}

\author{
Michelangelo Bin ${ }^{\mathrm{a}}$, Lorenzo Marconi $^{\mathrm{a}}$, Andrew R. Teel ${ }^{\mathrm{b}}$ \\ ${ }^{\text {a } C A S Y-D E I, ~ U n i v e r s i t y ~ o f ~ B o l o g n a, ~ B o l o g n a, ~ 40136, ~ I t a l y ~}$ \\ ${ }^{\mathrm{b}}$ Department of Electrical and Computer Engineering, University of California Santa Barbara, Santa Barbara, CA 93106, USA
}

\begin{abstract}
The problem of output regulation for general multivariable linear systems has been solved in the 70s, in the seminal works of Francis, Wonham and Davison, under the assumption that the reference signals and the disturbances acting on the system are generated by a known exogenous linear system (the exosystem). The regulator is designed to embed an internal model of the exosystem, which ensures that asymptotic regulation is maintained under arbitrary plant perturbations that do not destroy linearity and closed-loop stability. This robustness property, however, is inexorably lost whenever the internal model does not match exactly the exosystem. In this paper we endow the linear regulator with a discrete-time adaptive unit that adapts the regulator's internal model on the basis of the closed-loop evolution. Compared to existing approaches, adaptation here is cast as an identification problem, and the corresponding optimal predictor is designed independently from the underlying control system. This permits to separate stabilization and adaptation, thus naturally handling general non-square multivariable non minimum-phase plants. Closed-loop stability is guaranteed and, if the dimension of the internal model is large enough and a persistency of excitation condition is fulfilled, asymptotic regulation is achieved for references and disturbances generated by an unknown exosystem. Robustness to parametric uncertainties is inherited by the linear regulator and robustness to additional unmodeled disturbances is proved to hold.
\end{abstract}

Key words: Output Regulation; Linear Systems; Adaptive Systems; Internal Model; Identification for Control.

\section{Introduction}

The multivariable linear regulator, introduced by Francis, Wonham and Davison in $[1,2,3]$, boasts a very important robustness property relative to uncertainties in the plant: the regulation errors are ensured to vanish despite arbitrarily large perturbations in the plant dynamics as far as they do not destroy linearity and closed-loop stability. Roughly speaking, this robustness property is a consequence of the fact that if the closed-loop system is linear, and its origin is exponentially stable whenever the input is not present, its steady state is completely governed by the exosystem (interestingly, this is also true in infinite dimension [4]). As far as linearity and expo-

\footnotetext{
ऋ This paper was not presented at any IFAC meeting. Corresponding author Michelangelo Bin. Research supported by the European project AirBorne (ICT 780960), by AFOSR grant FA9550-15-1-0155, AFOSR grant AFOSR FA9550-181-0246 and NSF grant ECCS-1508757.

Email addresses: michelangelo.bin@unibo.it

(Michelangelo Bin), lorenzo.marconi@unibo.it (Lorenzo Marconi), teel@ece.ucsb.edu (Andrew R. Teel).
}

nential stability hold, the internal model embedded in the regulator is still able to generate the ideal "feedforward" control law that is needed to keep the regulation errors to zero at the steady state, and uncertainties in the plant just reflect into the right initialization of the internal model, which would be anyway unknown. As this intrinsic robustness is a consequence of the presence of an internal model of the exosystem, not surprisingly the linear regulator fails to guarantee asymptotic regulation as soon as the exosystem is not perfectly known. In other words, the linear regulator provides no robustness relative to arbitrarily small perturbations on the exosystem (for a more general treatise about robustness of regulation schemes the reader is referred to [5]).

The general problem of designing a regulator that ensures asymptotic regulation for linear systems in the presence of uncertainties in the exosystem is still open, and the existing results cover only limited classes of plants or exosystems. Single-input single-output (SISO) linear systems have been considered in [6,7] using adaptive observers. In the first work the order of the exosystem is known; in the second the knowledge of its 
upper bound is sufficient. In both the papers, though, the plant's matrices are assumed to be perfectly known, so as robustness relative to exosystems perturbations is traded for those relative to the plant. For what concerns adaptive designs for multivariable linear systems, strongly minimum-phase normal forms have been considered in [8], state-feedback tracking for more general linear systems has been studied in [9], and both continuous and discrete-time transfer functions have been considered in [10] and [11], in a more practice-oriented setting. Other approaches have been developed in the context of nonlinear systems. Nonlinear systems in output feedback form driven by uncertain linear exosystems have been considered for instance in $[12,13]$, where adaptive backstepping techniques are used. Nonlinear minimum-phase SISO normal forms have been considered in many papers. For example, in [14] an ad hoc adaptation algorithm is constructed based on Lyapunov arguments, in [15] adaptation is carried out by using the theory of adaptive observers, in [16] unknown linear exosystems are immersed into larger parameterless nonlinear exosystem whose dynamic is known and that can be dealt with in a nonlinear regulation setting. The same idea was applied to a class of uncertain nonlinear exosystems in $[17,18]$. Finally, in $[19,20,21]$ adaptation is cast as a system identification problem, and parameter estimation is performed by any continuous- or discrete-time algorithm satisfying some strong stability properties.

In this paper we consider the output regulation problem for general multivariable linear systems, with the reference signals and the disturbances that are generated by an unknown exosystem. We endow the linear regulator of [3] with a discrete-time identification unit which adapts the internal model on the basis of the closedloop measurable states. The identification algorithm implements a recursive least-squares scheme that enjoys strong stability properties with respect to an "optimal steady state" defined by the measured data. The regulator is designed to ensure the existence of a "temporary" steady state between two successive updates of the identifier, despite the possible wrong value of the estimated parameters. Even if the regulator errors do not vanish in this temporary steady state, the controlled plant still oscillates with the same modes of the exosystem, thus unveiling to the identifier the unknown frequency content of the external excitation. This, in turn, allows the identifier to eventually estimate the "right" parameters, no matter how "wrong" are the temporary steady states, as long as the dimension of the internal model is sufficiently large and a persistency of excitation condition is fulfilled. Moreover, the regulator corresponding to the "right" parameter has the internal model property, and it thus guarantees asymptotic regulation. In this respect, we observe that the proposed approach can be framed in a "dual control" perspective (see e.g. $[22,23]$ ), where the regulator plays the double role of inducing the right dynamics making the identification of the unknown parameters possible, and then stabilizing the "right" steady state when the parameter is correctly estimated. In Section 2 we present the problem formulation and the main standing assumptions. In Section 3 the proposed regulator is constructed and in Section 4 the main result on the closed-loop properties is given. The proof of the main result is presented in Section 5, while the proof of all the other auxiliary results is postponed to the appendix.

Notation: $\mathbb{R}, \mathbb{C}$ and $\mathbb{N}$ denote the sets of real, complex and natural numbers (0 included) and we let $\mathbb{R}_{>0}:=[0, \infty)$ and $\mathbb{R}_{>0}:=(0, \infty)$. We denote by $|\cdot|$ the vector or matrix 2 -norm. With $\mathcal{X}$ an Euclidean space we denote by $\mathbb{B}_{r}$ the open ball of radius $r \geq 0$ on $\mathcal{X}$. If $X \subset \mathcal{X}$ we let $|X|:=$ $\sup _{x \in X}|x|$ and, for $z \in \mathcal{X}$, we let $|z|_{X}:=\inf _{x \in X}|z-x|$ be the usual point-set distance. With $A, B \subset \mathbb{R}^{n}, n \in \mathbb{N}$, we let $A \backslash B:=\{a \in A: a \notin B\}$ and $A+B:=$ $\left\{a+b \in \mathbb{R}^{n}: a \in A, b \in B\right\}$. If $X \subset \mathcal{X}$ is a vector subspace, $X^{\perp}$ denotes its orthogonal complement. For a square matrix $A, \sigma(A)$ denotes the set of eigenvalues of $A$ and $\varphi_{A}(s)$ its characteristic polynomial. $\otimes$ denotes the Kronecker product of matrices. $\operatorname{Im} M$ denotes the column space of a matrix $M$. If $A_{1}, \ldots, A_{n}$ are matrices with the same number of columns we let $\operatorname{col}\left(A_{1}, \ldots, A_{n}\right)$ be their vertical concatenation. For a function $f: \mathbb{R}^{n} \rightarrow$ $\mathbb{R}$ and a vector field $g: \mathbb{R}^{n} \rightarrow \mathbb{R}^{n}, L_{g} f(x)$ denotes the Lie derivative of $f(\cdot)$ along $g(\cdot)$ computed at $x \in \mathbb{R}^{n}$. In this paper we consider hybrid systems of the form

$$
\mathcal{H}: \begin{cases}\dot{x}=F(x, u) & (x, u) \in C \\ x^{+}=G(x, u) & (x, u) \in D\end{cases}
$$

with state $x$ and input $u$ taking values in the Euclidean spaces $\mathcal{X}$ and $\mathcal{U}$, and where $F, G: \mathcal{X} \times \mathcal{U} \rightarrow \mathcal{X}$ denote the flow and jump maps and $C, D \subseteq \mathcal{X} \times \mathcal{U}$ the sets in which flows and jumps are allowed. We recall hereafter the main concepts and notations used in the paper to deal with systems of the kind (1) in the formalism of [24]; for further details the reader is referred to $[24,25]$. A compact hybrid time domain is a subset of $\mathbb{R}_{\geq 0} \times \mathbb{N}$ of the form $\mathcal{T}=\cup_{j=0}^{J-1}\left[t_{j}, t_{j+1}\right] \times\{j\}$ for some finite $J \in \mathbb{N}$ and $0=t_{0} \leq t_{1} \leq \cdots \leq t_{J} \in \mathbb{R}_{\geq 0}$. A set $\mathcal{T} \subset \mathbb{R}_{>0} \times \mathbb{N}$ is called a hybrid time domain if for each $(T, J) \in \mathbb{R}_{>0} \times \mathbb{N} \mathcal{T} \cap[0, T] \times\{1, \ldots, J\}$ is a compact hybrid time domain. If $(t, j),(s, i) \in \mathcal{T}$, we write $(t, j) \preceq(s, i)$ if $t+j \leq s+i$. For any $(t, j) \in \mathcal{T}$, we let $t_{j}=\inf _{t \in \mathbb{R}}(t, j) \in \mathcal{T}$ and $t^{j}=\sup _{t \in \mathbb{R}}(t, j) \in \mathcal{T}$. For brevity, we also let $\Gamma(\mathcal{T}):=\{(t, j) \in \mathcal{T}:(t, j+1) \in \mathcal{T}\}$ and, for $s \in \mathbb{R}_{\geq 0},\left.\mathcal{T}\right|_{\geq s}:=\{(t, j) \in \mathcal{T}: t+j \geq s\}$. If $\varphi$ is a hybrid arc, then we write $\varphi(t, j)^{+}$as a shortcut for $\varphi(t, j+1)$, whenever $(t, j) \in \Gamma(\varphi)$. A function $x: \mathcal{T} \rightarrow \mathcal{X}$ defined on a hybrid time domain $\mathcal{T}$ is called a hybrid arc if $x(\cdot, j)$ is locally absolutely continuous for each $j$. By borrowing the notation used in [25], a hybrid input is a hybrid arc that is locally essentially bounded and Lebesgue measurable. A solution pair [25] to (1) is a pair $(x, u)$, with $x$ a hybrid arc and $u$ a hybrid input, that satisfies such equations. We call 
a solution pair maximal if it cannot be extended further and complete if its time domain is unbounded. If $x: \operatorname{dom} x \rightarrow \mathcal{X}$ is a hybrid arc, we let $\limsup x:=$ $\lim \sup _{(t, j) \in \operatorname{dom} x, t+j \rightarrow \infty} x(t, j)$. If $u: \operatorname{dom} u \rightarrow \mathcal{U}$ is a hybrid input, for any $(t, j) \in \operatorname{dom} u$ we let $|u|_{(t, j)}:=$ $\max \left\{\operatorname{ess} \sup _{(s, i) \in \operatorname{dom} u / \Gamma(\operatorname{dom} u),(0,0) \preceq(s, i) \preceq(t, j)}|u(s, i)|\right.$, $\left.\sup _{(t, j) \in \Gamma(\operatorname{dom} u),(0,0) \preceq(s, i) \preceq(t, j)}|u(s, i)|\right\}$. We also let $|u|_{\infty}:=\lim \sup |u|_{(t, j)}$. When the flow and jump sets are omitted in the definition of a hybrid system they are intended to be the whole state space. If jumps of a variable are not specified in the equations then they are assumed to be jumps that do not change the state. Similarly, if flows of a variables are not specified, then they are assumed to be constant flows.

\section{Problem Formulation}

We consider linear systems of the form

$$
\begin{aligned}
& \dot{x}=A x+B u+P w+M \nu \\
& y=C x+Q w+N \nu
\end{aligned}
$$

where $x \in \mathbb{R}^{n}$ is the state, $u \in \mathbb{R}^{m}$ the control input, $y \in \mathbb{R}^{n_{y}}$ the measured outputs and $(w, \nu) \in \mathbb{R}^{n_{w}} \times \mathbb{R}^{n_{\nu}}$ represent exogenous signals acting on the system, such as references to be tracked and disturbances to be rejected. In particular, $w(t)$ represents the "modeled" part of the exogenous signals, and we suppose that it belongs to the family of solutions of an exosystem of the form

$$
\dot{w}=S w
$$

with unknown system matrix $S$ and with a dimension $n_{w} \in \mathbb{N}$ that is upperbounded by a known integer $d \in \mathbb{N}$. The signal $\nu(t)$, instead, is a bounded hybrid input representing unknown unmodeled disturbances, i.e. disturbances acting on the plant whose nature is not known and that are not supposed to be generated by any external process. We associate to (2) a further set of outputs $e \in \mathbb{R}^{n_{e}}, n_{e} \in \mathbb{N}$, defined as

$$
e=C_{e} x+Q_{e} w+N_{e} \nu
$$

We refer to the quantity $e$ as the regulation errors. They represent those outputs on which the effect of the exosystem must be ideally removed such as, for instance, tracking errors. More precisely, we seek an output feedback regulator of the form

$$
\begin{aligned}
& \dot{x}_{c} \in F_{c}\left(x_{c}, y\right) \quad\left(x_{c}, y\right) \in C_{c} \\
& x_{c}^{+} \in G_{c}\left(x_{c}, y\right) \quad\left(x_{c}, y\right) \in D_{c} \\
& u=\gamma\left(x_{c}, y\right)
\end{aligned}
$$

with state $x_{c}$ taking values in an Euclidean space $\mathcal{X}_{c}$, and where $C_{c}, D_{c} \subset \mathcal{X}$, such that all the solutions to the closed-loop system (2), (5) are bounded and it has the $\varepsilon$-approximate regulation property for some $\varepsilon>0$, i.e. any solution also satisfies

$$
\limsup |e| \leq \varepsilon
$$

We say that (2), (3), (5) has the asymptotic regulation property if it has the 0 -approximate regulation property. On the system (2), (3), (4) we make the following assumptions:

A1) The regulation errors are included in the measured output, i.e. $y=\operatorname{col}\left(e, y_{m}\right)$ for some $y_{m} \in \mathbb{R}^{n_{m}}, n_{m}:=$ $n_{y}-n_{e}$.

A2) $(A, B)$ is stabilizable, $(C, A)$ is detectable, $\operatorname{rank} B=$ $m \geq \operatorname{rank} C_{e}=n_{e}$.

A3) The solutions of (3) range in a compact set $W \subset$ $\mathbb{R}^{n_{w}}$ and $|W|$ is known.

A1 and A2 are close to being necessary if a robust asymptotic regulation result is sought. As a matter of fact, readability in the sense of [1] of $e$ from $y$ is proved in [1] to be necessary to obtain a structurally stable solution in the case in which (3) is known. If readability holds, on the other hand, we can always change coordinates to have A1 fulfilled. Furthermore, as in the classical solution of [3], we will augment the plant (2) with an internal model unit that is driven by the regulation errors. Then A2 turns out to be necessary to have stabilizability and detectability of the resulting cascade. Regarding A3, this assumption limits the size of the initial conditions of $w$ and requires $S$ to be stable though not typically Hurwitz. The set $W$ can be arbitrarily large as soon as $|W|$ is known. This latter quantity represents a constant that must be dominated by some control parameters. Thus, in this sense, the forthcoming result could also be rephrased by fixing the control parameters and adjusting the "admissible" $W$ accordingly.

\section{The Regulator Structure}

In this section we construct a regulator of the form (5) that guarantees closed-loop stability and, under suitable persistency of excitation conditions, has the $\varepsilon$-approximate regulation property with $\varepsilon$ that is proportional to limsup $|\nu|$. The regulator consists of two main blocks: the internal model block and the stabilizer (see Figure 1). The internal model block is itself composed of two subsystems: the internal model and the identifier. The internal model is a system driven by the regulation errors and it replicates the structure proposed by Davison in [3]. The spectrum of the internal model's system matrix is adapted by the identifier to match the modes of the unknown exosystem (3). The identifier is a discrete-time system built to solve asymptotically an optimization problem defined on the time 


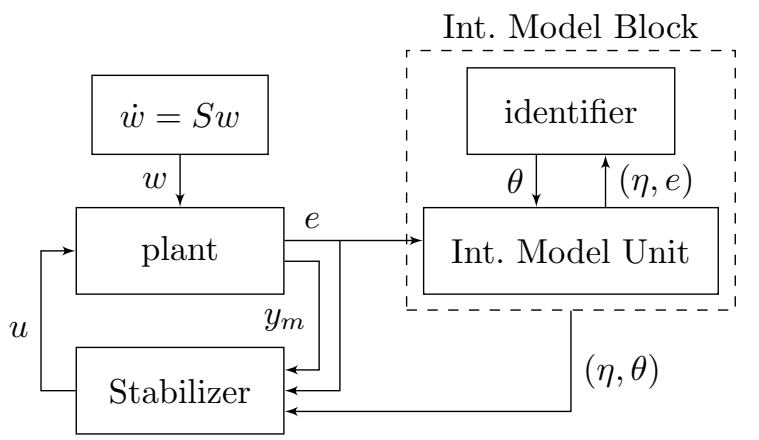

Figure 1. Block-diagram of the closed-loop system.

evolution of the state of the internal model and the regulation errors. Under suitable persistency of excitation conditions and if $\nu=0$, it turns out that the identifier optimal trajectory is uniquely determined and the corresponding internal model includes all the exosystem's modes, as long as asymptotic regulation is achievable. The stabilizer is a subsystem that processes all the measured signals and robustly stabilizes the cascade of the plant and the internal model block. In the rest of the section we detail all these three components.

\subsection{The Internal Model Unit}

With $d$ any known upper bound on $n_{w}$, the internal model unit is a system with state $\eta \in \mathbb{R}^{n_{e} d}$ satisfying the following equation

$$
\dot{\eta}=\Phi(\theta) \eta+G e
$$

where

$\Phi(\theta):=\left(\begin{array}{c}0_{n_{e}(d-1) \times n_{e}} I_{n_{e}(d-1)} \\ \theta^{T} \otimes I_{n_{e}}\end{array}\right) \quad G:=\left(\begin{array}{c}0_{n_{e}(d-1) \times n_{e}} \\ I_{n_{e}}\end{array}\right)$

and with $\theta \in \mathbb{R}^{d}$ that is a parameter adapted by the identifier. The characteristic polynomial of $\Phi(\theta)$ reads as

$$
\varphi_{\Phi(\theta)}(\lambda)=\left(\lambda^{d}-\theta_{d} \lambda^{d-1}-\cdots-\theta_{2} \lambda-\theta_{1}\right)^{n_{e}}
$$

so as, if $S$ were known, the internal model of [3] could be obtained by letting the components of $\theta$ be the coefficients of any polynomial that has the eigenvalues of $S$ as roots. We then let $\mathcal{Q}$ be the set

$$
\begin{gathered}
\mathcal{Q}:=\left\{\theta \in \mathbb{R}^{d}: \operatorname{rank}\left(\begin{array}{cr}
A-\lambda I & B \\
C_{e} & 0
\end{array}\right)<n+n_{e},\right. \\
\lambda \in \sigma(\Phi(\theta))\}
\end{gathered}
$$

and we pick a compact set satisfying

$$
\mathcal{E} \subset \mathbb{R}^{d} \backslash \mathcal{Q}
$$

The existence of a non-empty set $\mathcal{E}$ satisfying the above property, which in general has to be assumed, is in turn necessary for the solvability of the problem. As a matter of fact, as shown for instance in [26, Lem. 4.1], for the regulation problem to have a solution for a given exosystem $(3), \mathbb{R}^{n} \backslash \mathcal{Q}$ must contain at least one $\theta \in \mathbb{R}^{d}$ such that $\sigma(S)=\sigma(\Phi(\theta))$. Moreover, we have the following sufficient condition.

Lemma 1 Assume A1-A2 and suppose that the set of $\lambda \in \mathbb{C}$ for which the transfer function $C_{e}(A-\lambda I)^{-1} B$ loses rank is finite. Then for each $r \geq 0, \mathbb{R}^{d} \backslash\left(\mathcal{Q}+\mathbb{B}_{r}\right)$ is closed and not empty.

Lemma 1, whose proof is reported in Appendix A.1, implies that for any $r>0$ and any sufficiently large compact set $\Theta \subset \mathbb{R}^{d}$, the set $\left\{\theta \in \mathbb{R}^{d}:|\theta|_{\mathcal{Q}} \geq r\right\} \cap \Theta$ is compact and not empty, thus qualifying as a possible choice of $\mathcal{E}$. The definition of $\mathcal{E}$ is justified by the following fact:

Lemma 2 Let $\operatorname{rank} B \geq \operatorname{rank} C_{e}=n_{e}$. Then the pair

$$
\left(\left(\begin{array}{cc}
A & 0 \\
G C_{e} & \Phi(\theta)
\end{array}\right),\left(\begin{array}{l}
B \\
0
\end{array}\right)\right)
$$

is stabilizable/controllable for all $\theta \in \mathcal{E}$ if and only if $(A, B)$ is stabilizable/controllable. Moreover, the pair

$$
\left(\left(\begin{array}{cc}
C & 0 \\
0 & I
\end{array}\right),\left(\begin{array}{cc}
A & 0 \\
G C_{e} & \Phi(\theta)
\end{array}\right)\right)
$$

is detectable/observable for all $\theta \in \mathbb{R}^{d}$ if and only if $(C, A)$ is detectable/observable.

Lemma 2 is based on the PBH test [26], and its proof is a straightforward consequence of the definition of $\mathcal{Q}$ and it is thus omitted. In the forthcoming sections we will force $\theta$ to range in the set $\mathcal{E}$. Although this limits the number of internal models that can be eventually implemented, it also guarantees that the cascade of the plant and the internal model is stabilizable independently of the identifier trajectories.

\subsection{The Identifier}

The identifier subsystem is introduced in this section as a system built to solve a dynamic optimization problem defined on its inputs. Its definition is stated without any apparent link to the rest of the regulator, to which it will be interconnected only at the end of the section. 
The identifier measures two inputs at the jump times and it tries to infer a model relating the two inputs on the basis of the observed samples. The model is a linear regression of order $d$ and the parameter is chosen to minimize a cost function that weights a sum of historical prediction errors produced by the candidate model. For simplicity, in this paper we let the identifier to implement a recursive least squares identifier, nevertheless we remark that any other identification algorithm that enjoy similar properties of those stated in the following Proposition 1 and Lemma 4 can be used as well. At the end of the section we will interconnect the identifier with the internal model, obtaining a hybrid system that acts as a feedforward generator during flows and optimizes its internal model during jumps.

In the following we identify $\left(\mathbb{R}^{n_{e}}\right)^{d}$ with $\mathbb{R}^{n_{e} d}$ and, for each of its elements $p$, we let $p_{1}, \ldots, p_{d}$ denote the elements of $\mathbb{R}^{n_{e}}$ such that $p=\operatorname{col}\left(p_{1}, \ldots, p_{d}\right)$. Then we define the matrix

$$
\gamma(p):=\left(\begin{array}{llll}
p_{1} & p_{2} & \cdots & p_{d}
\end{array}\right)^{T} \in \mathbb{R}^{d \times n_{e}} .
$$

Let $\alpha$ and $\beta$ be hybrid inputs (see the notations paragraph) taking values in $\mathbb{R}^{n_{e} d}$ and $\mathbb{R}^{n_{e}}$ respectively. We define the identifier subsystem as a discrete-time system defined on the state space $\mathcal{Z} \times \mathbb{R}^{d}$, where $\mathcal{Z}:=\mathbb{R}^{d \times d} \times \mathbb{R}^{d}$, and with state $z:=(R, v) \in \mathcal{Z}$ and $\theta \in \mathbb{R}^{d}$ satisfying the following equations

$$
\begin{aligned}
& R^{+}=\mu R+\gamma(\alpha) \gamma(\alpha)^{T} \\
& v^{+}=\mu v+\gamma(\alpha) \beta \\
& \theta^{+} \in p_{\mathcal{E}}\left(R^{\dagger} v\right)
\end{aligned}
$$

with output $\theta$, where $\cdot \cdot^{\dagger}$ denotes the Moore-Penrose pseudoinverse, $\mu \in(0,1)$ is a design parameter and $p_{\mathcal{E}}(\cdot)$ is the projection map $\theta \mapsto p_{\mathcal{E}}(\theta):=\arg \inf _{\theta_{\mathcal{E}} \in \mathcal{E}}\left|\theta-\theta_{\mathcal{E}}\right|$. We endow $\mathcal{Z}$ with the norm $|(R, v)|:=\sqrt{|R|^{2}+|v|^{2}}$.

The identifier (9) is constructed to asymptotically find the "best" linear model relating the regressor input $\alpha$ and the input $\beta$. More precisely, we associate to (9) the prediction model $\hat{\beta}: \mathcal{E} \times \mathbb{R}^{n_{e} d} \rightarrow \mathbb{R}^{n_{e}}$ given by

$$
\hat{\beta}(\theta, \alpha):=\left(\theta^{T} \otimes I_{n_{e}}\right) \alpha=\sum_{i=1}^{d} \theta_{i} \alpha_{i}
$$

and the corresponding prediction error $\varepsilon: \mathcal{E} \times \mathbb{R}^{n_{e} d} \times$ $\mathbb{R}^{n_{e}} \rightarrow \mathbb{R}^{n_{e}}$ given by

$$
\varepsilon(\theta, \alpha, \beta):=\beta-\hat{\beta}(\theta, \alpha)=\beta-\left(\theta^{T} \otimes I_{n_{e}}\right) \alpha .
$$

For fixed $\theta$, the prediction model $\hat{\beta}(\theta, \alpha)$ represents the identifier's guess of $\beta$ given $\alpha$. The identifier is constructed to choose $\theta$ so that the guess $\hat{\beta}(\theta, \alpha)$ is the best possible among all those producible by $d$-dimensional linear models of the form (10). Here "best" is defined relative to a cost function that weights the prediction performance of the candidate models on all the historical data. More precisely, with $\mathcal{A}\left(\mathbb{R}^{d}, \mathbb{R}_{\geq 0}\right)$ the set of functions $\mathbb{R}^{d} \rightarrow \mathbb{R}_{\geq 0}$, we associate to each input $(\alpha, \beta)$ a function $\mathcal{J}_{\alpha, \beta}: \operatorname{dom}(\alpha, \beta) \rightarrow \mathcal{A}\left(\mathbb{R}^{d}, \mathbb{R}_{\geq 0}\right)$ defined by

$$
\mathcal{J}_{\alpha, \beta}(t, j)(\theta):=\sum_{i=0}^{j-1} \mu^{j-i-1}\left|\varepsilon\left(\theta, \alpha\left(t^{i}, i\right), \beta\left(t^{i}, i\right)\right)\right|^{2}
$$

At each $(t, j) \in \operatorname{dom}(\alpha, \beta)$, and for fixed $\theta \in \mathbb{R}^{d}$, the function (11) is a weighted sum of the squares ${ }^{1}$ of the prediction errors of the model (10) corresponding to $\theta$ evaluated during the jump times. The control parameter $\mu \in(0,1)$ plays the role of a forgetting factor, making past samples less important in the sum. At a given $(t, j) \in \operatorname{dom}(\alpha, \beta)$, the best linear model is the one given by (10) with $\theta$ minimizing $\mathcal{J}_{\alpha, \beta}(t, j)$. We associate to (11) the following (set-valued) map

$$
\theta_{\alpha, \beta}^{\circ}(t, j):=\underset{\theta \in \mathbb{R}^{d}}{\arg \min } \mathcal{J}_{\alpha, \beta}(t, j)(\theta),
$$

whose value at each $(t, j)$, contains the "optimal" parameters $\theta$ that minimize (11).

The intuition behind the definition of the identifier (9), in relation to the minimization problem (11), resides in the fact that the optimal trajectory (12) can be proved to satisfy

$$
\theta_{\alpha, \beta}^{\circ}(t, j)=\left\{\theta \in \mathbb{R}^{d}: R^{\star}(t, j) \theta=v^{\star}(t, j)\right\},
$$

where we defined

$$
\begin{aligned}
R^{\star}(t, j) & :=\sum_{i=0}^{j-1} \mu^{j-i-1} \gamma\left(\alpha\left(t^{i}, i\right)\right) \gamma\left(\alpha\left(t^{i}, i\right)\right)^{T} \\
v^{\star}(t, j) & :=\sum_{i=0}^{j-1} \mu^{j-i-1} \gamma\left(\alpha\left(t^{i}, i\right)\right) \beta\left(t^{i}, i\right) .
\end{aligned}
$$

As stated in more general terms in the forthcoming proposition, it can be shown that $z^{\star}:=\left(R^{\star}, v^{\star}\right)$ is a solution to the subsystem $z$ of (9) which is also (robustly) asymptotically stable. It is worth noting, moreover, that while the definition of (11) requires in principle the knowledge of an unbounded number of samples, in view of (14), the information that is necessary to define (13) can be encoded in the finite dimensional quantities $R^{\star}$ and $v^{\star}$, and this permits to track the optimal trajectory (12) with a finite-dimensional system (as it is (9)).

\footnotetext{
1 For this reason we call the identifier (9) a (weighted) leastsquares identifier.
} 
When we will interconnect the identifier and the internal model, the inputs $(\alpha, \beta)$ will be set to some functions of the state $\eta$ and the regulation errors $e$. These signals, in turn, carry some "ideal" information about $w(t)$, that is useful to infer the right model, corrupted by some additional disturbances given by transitory artifacts and residual noise dependent on $\nu$. To take into account this situation in the characterization of the properties of the identifier (9), we consider inputs $(\alpha, \beta)$ given by $\alpha=\alpha^{\star}+\delta_{\alpha}, \beta=\beta^{\star}+\delta_{\beta}$, with $\left(\alpha^{\star}, \beta^{\star}\right)$ an ideal input and $\delta=\left(\delta_{\alpha}, \delta_{\beta}\right)$ an additive disturbance. The following proposition expresses the optimality of the identifier (9) with respect to the cost function $\mathcal{J}_{\alpha^{\star}, \beta^{\star}}$ when $\delta=0$ and the robust stability properties of the optimal trajectory in case of $\delta \neq 0$.

\section{Proposition 1 The following hold:}

(1) For each $z_{1}:=\left(R_{1}, v_{1}\right), z_{2}:=\left(R_{2}, v_{2}\right) \in \mathcal{Z}$ and each $\left(\alpha_{1}, \beta_{1}\right),\left(\alpha_{2}, \beta_{2}\right) \in \mathbb{R}^{d n_{e}} \times \mathbb{R}^{n_{e}}$ there exists $\rho>0$ such that, with $\left(\delta_{\alpha}, \delta_{\beta}\right):=\left(\alpha_{1}, \beta_{1}\right)-\left(\alpha_{2}, \beta_{2}\right)$, it holds that

$$
\left|z_{1}^{+}-z_{2}^{+}\right|^{2} \leq \mu^{2}\left|z_{1}-z_{2}\right|^{2}+\rho\left|\left(\delta_{\alpha}, \delta_{\beta}\right)\right|^{2}
$$

where, for $i=1,2$, we let $z_{i}^{+}:=\left(\mu R_{i}+\right.$ $\left.\gamma\left(\alpha_{i}\right) \gamma\left(\alpha_{i}\right)^{T}, \mu v_{i}+\gamma\left(\alpha_{i}\right) \beta_{i}\right)$

(2) For each hybrid input $\left(\alpha^{\star}, \beta^{\star}\right): \operatorname{dom}\left(\alpha^{\star}, \beta^{\star}\right) \rightarrow$ $\mathbb{R}^{d n_{e}} \times \mathbb{R}^{n_{e}}$ there exists $z^{\star}:=\left(R^{\star}, v^{\star}\right): \operatorname{dom}\left(\alpha^{\star}, \beta^{\star}\right)$ $\rightarrow \mathcal{Z}$ such that $\left(z^{\star},\left(\alpha^{\star}, \beta^{\star}\right)\right)$ is a solution pair to the subsystem $z$ of $(9)$ and the corresponding "unconstrained output" $\theta_{\text {un }}^{\star}:=\left(R^{\star}\right)^{\dagger} v^{\star}$ satisfies

$$
\theta_{u n}^{\star}(t, j) \in \theta_{\alpha^{\star}, \beta^{\star}}^{\circ}(t, j) .
$$

Although Proposition 1 guarantees that for every input $(\alpha, \beta)=\left(\alpha^{\star}, \beta^{\star}\right)+\left(\delta_{\alpha}, \delta_{\beta}\right)$ there exists a "optimal trajectory" $\left(z^{\star}, \theta_{u n}^{\star}\right)$ such that, for any selection $\theta^{\star}$ of $p_{\mathcal{E}}\left(\theta_{u n}^{\star}\right),\left(\left(z^{\star}, \theta^{\star}\right),\left(\alpha^{\star}, \beta^{\star}\right)\right)$ solves $(9)$ and is asymptotic stable (with $\delta=0$ ), it is not in general true that $z \rightarrow z^{\star}$ implies $\theta \rightarrow \theta^{\star}$, due to the pseudoinverse operator that is not, in general, continuous. To recover this "detectability" property, as well as to ensure single-valuedness of (12), we associate to the input $\alpha$ the following persistency of excitation condition.

Definition 1 With $J \in \mathbb{N}$ and $\epsilon>0$, a complete hybrid input $\alpha: \operatorname{dom} \alpha \rightarrow \mathbb{R}^{n_{e} d}$ is said to be $(J, \epsilon)$-persistently exciting if, for all integers $j \geq J$

$$
\min \sigma\left(\sum_{i=0}^{j-1} \mu^{j-i-1} \gamma\left(\alpha\left(t^{i}, i\right)\right) \gamma\left(\alpha\left(t^{i}, i\right)\right)^{T}\right) \geq \epsilon
$$

In the following we will often abbreviate " $(J, \epsilon)$ persistently exciting" with " $(J, \epsilon)-\mathrm{PE}$ ". Lemma 3 relates persistency of excitation of $\alpha$ with that of $\alpha^{\star}$ and, thus, with single-valuedness of the map $\theta_{\alpha^{\star}, \beta^{\star}}^{\circ}(t, j)$, when the disturbance $\delta_{\alpha}$ is small enough at the jump times. Lemma 4, instead, links persistency of excitation and "detectability" from the output $\theta$.

Lemma 3 Let $\alpha, \alpha^{\star}$ and $\delta_{\alpha}$ be bounded hybrid inputs defined over the same time domain and such that $\alpha=$ $\alpha^{\star}+\delta_{\alpha}$. Then for any $\epsilon>0$ there exists $\bar{\delta}>0$ such that, if $\alpha$ is $(J, \epsilon)-P E$ for some $J \in \mathbb{N}$ and $\left|\delta_{\alpha}\left(t^{j}, j\right)\right| \leq \bar{\delta}$ for all $j \geq J$, then there exists $\left(J^{\prime}, \epsilon^{\prime}\right) \in \mathbb{N} \times \mathbb{R}_{>0}$ such that $\alpha^{\star}$ is $\left(J^{\prime}, \epsilon^{\prime}\right)-P E$. Moreover, $\theta_{\alpha^{\star}, \beta^{\star}}^{\circ}(t, j)$ is a singleton for all $(t, j) \in \operatorname{dom} \alpha^{\star}$ such that $j \geq J^{\prime}$.

Lemma 4 Let $\left(\left(z_{1}, \theta_{1}\right),\left(\alpha_{1}, \beta_{1}\right)\right)$ and $\left(\left(z_{2}, \theta_{2}\right),\left(\alpha_{2}, \beta_{2}\right)\right)$ be solution pairs to (9) with the same time domain. Suppose that, for $i=1,2, \alpha_{i}$ is $\left(J_{i}, \epsilon_{i}\right)-P E$, for some $\left(J_{i}, \epsilon_{i}\right) \in$ $\mathbb{N} \times \mathbb{R}_{>0}$. Then there exist $J \in \mathbb{N}$ and $a \geq 0$ such that

$$
\left|\theta_{1}(t, j)-\theta_{2}(t, j)\right|^{2} \leq a\left|z_{1}(t, j)-z_{2}(t, j)\right|^{2},
$$

for all $(t, j) \in \operatorname{dom} z$ satisfying $j \geq J$.

With the above definitions in mind, we interconnect the identifier (9) and the internal model (6) by letting $\theta$ in (6) be the same state variable of (9) and by letting in (9) $\alpha=\eta$ and $\beta=G^{T} \dot{\eta}=\dot{\eta}_{d}=\left(\theta^{T} \otimes I_{n_{e}}\right) \eta+e$, i.e.

$$
\begin{aligned}
R^{+} & =\mu R+\gamma(\eta) \gamma(\eta)^{T} \\
v^{+} & =\mu v+\gamma(\eta)\left(\left(\theta^{T} \otimes I_{n_{e}}\right) \eta+e\right) \\
\theta^{+} & \in p_{\mathcal{E}}\left(R^{\dagger} v\right) .
\end{aligned}
$$

\subsection{The Stabilizer}

The stabilizer is defined as the composition of a continuous-time output feedback controller for the cascade $(x, \eta, z)$ and a clock subsystem that activates the update law of the identifier. It reads as follows:

$$
\begin{gathered}
\left\{\begin{array}{l}
\dot{\tau}=1 \\
\dot{\xi}=H_{\xi}(\theta) \xi+H_{y}(\theta) y+H_{\eta}(\theta) \eta \\
(\tau, \xi, y, \eta) \in[0, \overline{\mathrm{T}}] \times \mathbb{R}^{n_{\xi}+n_{y}+n_{e} d}
\end{array}\right. \\
\left\{\begin{array}{l}
\tau^{+}=0 \\
\xi^{+}=
\end{array}\right. \\
\text {with state }(\tau, \xi) \in \mathbb{R} \times \mathbb{R}^{n_{\xi}}, n_{\xi} \in \mathbb{N}, \text { and output } \\
u=D_{\xi}(\theta) \xi+D_{y}(\theta) y+D_{\eta}(\theta) \eta .
\end{gathered}
$$

The parameters $\underline{\mathrm{T}}, \overline{\mathrm{T}}>0$ constrain the jump times. While $\underline{T}$ must be taken sufficiently large to achieve 
closed-loop stability (see Theorem 1 ), $\overline{\mathrm{T}}$ is only constrained to be larger or equal to $\underline{T}$. In this way the update law can be triggered with non-periodic timing strategies in the limits of the stability constraints. The subsystem $\xi$ is instead a continuous-time system that is designed to stabilize the closed-loop system during flows and with $w=0$. More precisely, (18)-(19) is designed so that:

P1. $H_{\xi}, H_{y}, H_{\eta}, D_{\xi}, D_{y}$ and $D_{\eta}$ are locally Lipschitz functions of $\theta$.

P2. The matrix

$$
F(\theta)=\left(\begin{array}{ccc}
A+B D_{y}(\theta) C & B D_{\eta}(\theta) & B D_{\xi}(\theta) \\
G C_{e} & \Phi(\theta) & 0 \\
H_{y}(\theta) C & H_{\eta}(\theta) & H_{\xi}(\theta)
\end{array}\right)
$$

is Hurwitz for all $\theta \in \mathcal{E}$.

We remark that, in view of Lemma 2, P1 and P2 always can be achieved. As a matter of fact, $\theta$ is available for feedback and the only matrix in the equations of $(x, \eta)$ that depends on $\theta$ is $\Phi(\theta)$, whose dependency is smooth.

\subsection{The Overall Regulator}

The overall regulator, obtained by interconnecting the subsystems (6), (17), (18), (19), is thus a hybrid system described by the following equations

$$
\begin{aligned}
& \left\{\begin{array}{l}
\dot{\tau}=1 \\
\dot{\eta}=\Phi(\theta) \eta+G e \\
\dot{R}=0, \dot{v}=0, \dot{\theta}=0 \\
\dot{\xi}=H_{\xi}(\theta) \xi+H_{y}(\theta) y+H_{\eta}(\theta) \eta
\end{array}\right. \\
& \quad \begin{array}{l}
(\tau, \eta, R, v, \theta, \xi, y) \in[0, \overline{\mathrm{T}}] \times \mathbb{R}^{n_{e} d} \times \mathcal{Z} \times \mathbb{R}^{d+n_{\xi}+n_{y}} \\
\tau^{+}=0 \\
\eta^{+}=\eta \\
R^{+}=\mu R+\gamma(\eta) \gamma(\eta)^{T} \\
v^{+}=\mu v+\gamma(\eta)\left(\left(\theta^{T} \otimes I_{n_{e}}\right) \eta+e\right) \\
\theta^{+} \in p_{\mathcal{E}}\left(R^{\dagger} v\right) \\
\xi^{+}=\xi \\
\quad(\tau, \eta, R, v, \theta, \xi, y) \in[\underline{\mathrm{T}}, \overline{\mathrm{T}}] \times \mathbb{R}^{n_{e} d} \times \mathcal{Z} \times \mathbb{R}^{d+n_{\xi}+n_{y}}
\end{array}
\end{aligned}
$$

with input $y$ and with output

$$
u=D_{\xi}(\theta) \xi+D_{y}(\theta) y+D_{\eta}(\theta) \eta .
$$

\section{Main Result}

We let for convenience $\mathbf{w}:=(w, \tau), \chi:=(x, \eta, \xi)$ and

$$
s_{\mathbf{w}}(\mathbf{w}):=\operatorname{col}(S w, 1), \quad g_{\mathbf{w}}(\mathbf{w}):=\operatorname{col}(w, 0)
$$

$$
\begin{aligned}
G_{z}(z, \chi):=\left\{\mu R+\gamma(\eta) \gamma(\eta)^{T}\right\} \\
\quad \times\left\{\mu v+\gamma(\eta)\left(\left(\theta^{T} \otimes I_{n_{e}}\right) \eta+e\right)\right\} \\
E(\theta):=\operatorname{col}\left(P+B D_{y}(\theta) Q, G Q_{e}, H_{y}(\theta) Q\right) \\
L(\theta):=\operatorname{col}\left(M+B D_{y}(\theta) R, G N_{e}, H_{y}(\theta) N\right) .
\end{aligned}
$$

Then, the closed loop system given by (2), (21), (22) reads as follows

$$
\left\{\begin{array} { l } 
{ \dot { \mathbf { w } } = s _ { \mathbf { w } } ( \mathbf { w } ) } \\
{ \dot { \chi } = F ( \theta ) \chi + E ( \theta ) w + L ( \theta ) \nu } \\
{ \dot { z } = 0 } \\
{ \dot { \theta } = 0 }
\end{array} \quad \left\{\begin{array}{l}
\mathbf{w}^{+}=g_{\mathbf{w}}(\mathbf{w}) \\
\chi^{+}=\chi \\
z^{+}=G_{z}(z, \chi) \\
\theta^{+} \in p_{\mathcal{E}}\left(R^{\dagger} v\right)
\end{array}\right.\right.
$$

with flow and jump sets given by $\mathcal{C}:=W \times[0, \overline{\mathrm{T}}] \times$ $\mathbb{R}^{n_{\chi}} \times \mathcal{Z} \times \mathbb{R}^{d} \times \mathcal{N}$ and $\mathcal{D}:=W \times[\underline{\mathrm{T}}, \overline{\mathrm{T}}] \times \mathbb{R}^{n_{\chi}} \times \mathcal{Z} \times$ $\mathbb{R}^{d} \times \mathcal{N}$, being $n_{\chi}:=n_{x}+n_{e} d+n_{\xi}$ and $\mathcal{N} \subset \mathbb{R}^{n_{\nu}}$ an arbitrarily large compact set. In the definition of $\mathcal{C}$ and $\mathcal{D}$ we restricted the flow and jump sets of $(w, \nu)$ to the compact set $W \times \mathcal{N}$. In this way we consider only solutions for which $w(t) \in W$ and $\nu(t) \in \mathcal{N}$. Since $W$ is assumed to be forward invariant for the exosystem (3), we maintain completeness of the solutions for all inputs $\nu$ satisfying $\nu(t, j) \in \mathcal{N}$.

As long as $n_{w} \leq d$, the Cayley-Hamilton Theorem guarantees the existence of $\omega \in \mathbb{R}^{d}$ such that the exosystem's system matrix $S$ satisfies

$$
S^{d}-\omega_{d} S^{d-1}-\cdots-\omega_{2} S-\omega_{1} I=0 .
$$

As mentioned in Section 3.1, if the internal model unit (6) is implemented with $\theta=\omega$, for any $\omega$ for which (24) holds, then asymptotic regulation is achieved. As we constrained $\theta$ to range in $\mathcal{E}$, we will eventually rely on the following assumption:

A4) There exists $\omega \in \mathcal{E}$ such that (24) holds.

The following theorem, which is the main result of the paper, states the main asymptotic properties of the proposed regulator.

Theorem 1 Assume A1-A3 and let (18) be chosen such that $P 1$ and $P 2$ hold. Then there exists $\underline{T}_{1}^{\star}$, such that if $\underline{\mathrm{T}} \geq \mathrm{T}_{1}^{\star}$, all the solutions of $(23)$ are bounded. If in addition A4 holds, for any $\epsilon>0$ there exist $\mathrm{T}_{2}^{\star} \geq \underline{\mathrm{T}}_{1}^{\star}$ and $\bar{\nu}, c \geq 0$ such that, if $\underline{\mathrm{T}} \geq \underline{\mathrm{T}}_{2}^{\star}$, for each complete solution pair to (23) for which $\eta$ is $(J, \epsilon)-P E$, for some $J \in \mathbb{N}$, and $|\nu|_{\infty} \leq \bar{\nu}$ the following holds

$$
\limsup |e| \leq c \limsup |\nu| \text {. }
$$

With reference to the proof of Theorem 1, we note that boundedness of the trajectories is obtained if $\mathrm{T}$ is larger than a quantity that depends only on the closed-loop 
system's data and that can be fixed after the regulator is designed. The bound (25) is instead more complex. It is in fact a property guaranteed just along the trajectories for which $\eta$ is $(J, \epsilon)-\mathrm{PE}$, for some $(J, \epsilon) \in \mathbb{N} \times \mathbb{R}_{>0}$, and only if $T$ is larger and limsup $|\nu|$ is smaller than constants that, in general, depend on $\epsilon$. Therefore the bound (25) is local in $\nu$, with the same constants, though, that work for any trajectory for which $\eta$ is $\left(J^{\prime}, \epsilon^{\prime}\right)$-PE with $J^{\prime} \in \mathbb{N}$ and $\epsilon^{\prime} \geq \epsilon$. We also remark that there is no uniformity in the convergence (25), as the convergence rate strongly depends on the particular $J$ for which the $(J, \epsilon)$-PE condition holds. This, however, matches with the intuition that the correct adaptation can take place only after the input signal to the identifier becomes sufficiently informative. Therefore, uniformity in the choice of $\underline{T}_{2}^{\star}$ and in the convergence (25) is possible only inside the class of solutions to the closed-loop system that are $(J, \epsilon)-\mathrm{PE}$ with the same $J$ and $\epsilon$. Finally we note that if $\nu=0$, i.e. if no unmodeled disturbances are present, then (25) implies asymptotic regulation, i.e. $e(t, j) \rightarrow 0$.

\section{$5 \quad$ Proof of Theorem 1}

The existence of $\underline{T}_{1}^{\star}$ such that for $\underline{T} \geq \underline{T}_{1}^{\star}$ the maximal trajectories of (23) are complete and bounded follows from standard "slow-switching" arguments (see for instance [27]) once noted that $F(\theta)$ and $E(\theta)$ are bounded uniformly in $\theta$ and boundedness of $\eta$ implies those of $z$. In proving the second claim, we articulate the discussion in the following 4 points.

\section{1) Quasi steady state of the stabilized cascade $\chi$}

We prove now that during the flow intervals the system $\chi$ evolves towards a "quasi" steady state determined by $w$ and parametrized by $\theta$. As $F(\theta)$ is Hurwitz for each $\theta \in \mathcal{E}$, there exist Lipschitz maps $P: \mathcal{E} \rightarrow \mathbb{R}^{n_{\chi} \times n_{\chi}}$ and $\Pi: \mathcal{E} \rightarrow \mathbb{R}^{n_{\chi} \times n_{w}}$, with $P(\cdot)$ having symmetric and positive definite values, that are point-wise solutions to

$$
\begin{aligned}
& F(\theta)^{T} P(\theta)+P(\theta) F(\theta)=-I_{n_{\chi}} \\
& \Pi(\theta) S=F(\theta) \Pi(\theta)+E(\theta) .
\end{aligned}
$$

Define the function

$$
V(w, \chi, z, \theta):=(\chi-\Pi(\theta) w)^{T} P(\theta)(\chi-\Pi(\theta) w) .
$$

Then, by letting $\underline{\sigma}:=\min \{\lambda \in \mathbb{R}: \lambda \in \sigma(P(\theta)), \theta \in \mathcal{E}\}$ and $\bar{\sigma}:=\max \{\lambda \in \mathbb{R}: \lambda \in \sigma(P(\theta)), \theta \in \mathcal{E}\}$, simple computations show that $V$ fulfills $\underline{\sigma}|\chi-\Pi(\theta) w|^{2} \leq$ $V(w, \chi, z) \leq \bar{\sigma}|\chi-\Pi(\theta) w|^{2}$ in the whole state space and

$$
L_{\mathcal{F}} V(w, \chi, z, \theta) \leq-\lambda V(w, \chi, z, \theta)+r_{0}|\nu|^{2}
$$

for all $(w, \chi, z, \theta)$ such that $(\mathbf{w}, \chi, z, \theta) \in \mathcal{C}$, with $\mathcal{F}:=$ $(S w, F(\theta) \chi+E(\theta) w+L(\theta) \nu, 0)$ and with $\lambda>0$. On the other hand, for all $(\mathbf{w}, \chi, z, \theta) \in \mathcal{D}$, we obtain

$$
\begin{aligned}
V\left(w^{+}, \chi^{+}, z^{+}\right. & \left., \theta^{+}\right) \leq \bar{\sigma}\left|\chi-\Pi\left(\theta^{+}\right)\right|^{2} \\
& \leq \bar{\sigma}\left(|\chi-\Pi(\theta) w|^{2}+\left|\Pi(\theta) w-\Pi\left(\theta^{+}\right) w\right|^{2}\right) \\
& \leq r_{1} V(w, \chi, z, \theta)+r_{2}\left|\theta-\theta^{+}\right|^{2}
\end{aligned}
$$

with $r_{1}=\bar{\sigma} / \underline{\sigma}$ and with $r_{2}>0$ properly chosen by using the fact that $w \in W, \theta^{+} \in \mathcal{E}$ and $\Pi(\cdot)$ is Lipschitz on $\mathcal{E}$.

\section{2) Properties of the identifier}

We show now that if the flow is long enough the distance of $\chi$ to its quasi steady state at jump times, where adaptation takes place, is sufficiently small to conclude that if $\eta$ is persistently exciting, then the identification problem associated to the steady-state signals has an unique optimum. Let $\Pi_{x}(\theta) \in \mathbb{R}^{n_{x} \times n_{w}}, \Pi_{\eta}(\theta) \in$ $\mathbb{R}^{n_{\eta} \times n_{w}}$ and $\Pi_{\xi}(\theta) \in \mathbb{R}^{n_{\xi} \times n_{w}}$ be such that $\Pi(\theta)=$ $\operatorname{col}\left(\Pi_{x}(\theta), \Pi_{\eta}(\theta), \Pi_{\xi}(\theta)\right)$ and, for $i=1, \ldots, d$, let $\Pi_{\eta_{i}}(\theta)$ be such that $\Pi_{\eta}(\theta)=\operatorname{col}\left(\Pi_{\eta_{1}}(\theta), \ldots, \Pi_{\eta_{d}}(\theta)\right)$. As a consequence of the structure of the matrix $\Phi(\theta)$, we have

$$
\left\{\begin{array}{l}
\Pi_{\eta_{i}}(\theta) S=\Pi_{\eta_{i+1}}(\theta), \quad i=1, \ldots, d-1 \\
\Pi_{\eta_{d}}(\theta) S=\left(\theta^{T} \otimes I_{n_{e}}\right) \Pi_{\eta}(\theta)+\Pi_{e}(\theta)
\end{array}\right.
$$

with $\Pi_{e}(\theta):=C_{e} \Pi_{x}(\theta)+Q_{e}$. Equation (29) also yields

$$
\begin{aligned}
\eta & =\Pi_{\eta}(\theta) w+\delta_{\alpha} \\
\left(\theta^{T} \otimes I_{n_{e}}\right) \eta+e & =\Pi_{\eta_{d}}(\theta) S w+\delta_{\beta}
\end{aligned}
$$

where $\delta_{\alpha}:=\eta-\Pi_{\eta}(\theta) w$ and $\delta_{\beta}:=\left(\theta^{T} \otimes I_{n_{e}}\right)(\eta-$ $\left.\Pi_{\eta}(\theta) w\right)+\left(e-\Pi_{e}(\theta) w\right)$ that satisfy

$$
\left|\left(\delta_{\alpha}, \delta_{\beta}\right)\right|^{2} \leq r_{3} V(w, \chi, z, \theta)+r_{4}|\nu|^{2}
$$

with $r_{3}:=\left(1+\left|C_{e}\right|^{2}+|\mathcal{E}|^{2 n_{e}}\right) / \underline{\sigma}$ and $r_{4}:=\left|N_{e}\right|^{2}$. The identifier subsystem can be thus seen as a system with input $(\alpha, \beta)=\left(\alpha^{\star}+\delta_{\alpha}, \beta^{\star}+\delta_{\beta}\right)$, where $\left(\alpha^{\star}, \beta^{\star}\right)=\left(\Pi_{\eta}(\theta) w, \Pi_{\eta_{d}}(\theta) S w\right)$ and $\left(\delta_{\alpha}, \delta_{\beta}\right)$ defined as before. This yields two consequences:

1) Single-valued Optimum: As $\nu(t, j) \in \mathcal{N}$ for each solution pair to (23), and $\lambda$ and $r_{1}$ in (27)-(28) are constants, we can assume without loss of generality that $\underline{T}_{1}^{\star}$ is chosen large enough so that $(27)-(28)$ can be turned, by using standard average dwell-time conditions $[28,29]$, to a ISS-Lyapunov function [30]. This in turn implies the existence of a $\Delta_{0}>0$, depending on $\mathcal{E}$ and $\mathcal{N}$, such that, for each solution pair $((\mathbf{w}, \chi, z, \theta), \nu)$ to (23) there exists $\bar{s}_{1}>0$ such that $|\chi(t, j)| \leq \Delta_{0}$ for all $\left.(t, j) \in \operatorname{dom}(\mathbf{w}, \chi, z, \theta)\right|_{\geq \bar{s}_{1}}$. Thus, in particular there exists $\Delta_{1}>0$ such that $|(\alpha(t, j), \beta(t, j))|=$ $\left|\left(\eta(t, j),\left(\theta(t, j)^{T} \otimes I_{n_{e}}\right) \eta(t, j)+e(t, j)\right)\right| \leq \Delta_{1}$ for all $\left.(t, j) \in \operatorname{dom}(\mathbf{w}, \chi, z, \theta)\right|_{\geq \bar{s}_{1}}$. Also, as $W$ and $\mathcal{E}$ are compact, there exists $\Delta_{2}>0$ such that $\left|\left(\alpha^{\star}(t, j), \beta^{\star}(t, j)\right)\right|=$ $\left|\left(\Pi_{\eta}(\theta(t, j)) w(t, j), \Pi_{\eta_{d}}(\theta(t, j)) S w(t, j)\right)\right| \leq \Delta_{2}$ for all $(t, j) \in \operatorname{dom}(\mathbf{w}, \chi, z, \theta)$. Suppose that $\alpha=\eta$ is $(J, \epsilon)-\mathrm{PE}$, 
for some $(J, \epsilon) \in \mathbb{N} \times \mathbb{R}_{\geq 0}$. Then, in view of Lemma 3 , there exist $\left(J^{\prime}, \epsilon^{\prime}\right) \in \mathbb{N} \times \mathbb{R}_{>0}$ and $\bar{\delta}>0$, depending on $\mathcal{E}, \mathcal{N}$ and $\epsilon$, such that $\left|\left(\delta_{\alpha}\left(t^{j}, j\right), \delta_{\beta}\left(t^{j}, j\right)\right)\right| \leq \bar{\delta}$ for all $j \geq J$ implies that $\alpha^{\star}$ is $\left(J^{\prime}, \epsilon^{\prime}\right)$-PE. Equation $(27)$ gives

$V\left(w\left(t^{j}, j\right), \chi\left(t^{j}, j\right), z\left(t^{j}, j\right), \theta\left(t^{j}, j\right)\right) \leq \bar{V} e^{-\lambda \underline{T}}+r_{0}|\nu|_{\infty}^{2} / \lambda$

for all $j \in \mathbb{N}$ such that $t^{j}+j \geq \bar{s}_{1}$ and with $\bar{V}:=$ $\bar{\sigma}\left(\Delta_{0}^{2}+|\Pi(\mathcal{E})|^{2}|W|^{2}\right)$. Therefore, as long as

$$
\begin{aligned}
& |\nu|_{\infty} \leq \bar{\nu}:=\bar{\delta} \sqrt{\frac{1}{3} \max \left\{\frac{\lambda}{r_{0} r_{3}}, \frac{1}{r_{4}}\right\}} \\
& \underline{\mathrm{T}} \geq \underline{\mathrm{T}}_{\epsilon}^{\star}:=\max \left\{\underline{\mathrm{T}}_{1}^{\star},(1 / \lambda) \log \left(3 \bar{V} r_{3} / \bar{\delta}^{2}\right)\right\}
\end{aligned}
$$

then (30) implies that every solution pair $((\mathbf{w}, \chi, z, \theta), \nu)$ to (23) with $\underline{\mathrm{T}} \geq \underline{\mathrm{T}}_{\epsilon}^{\star}$ for which $\eta$ is $(J, \epsilon)$-PE fulfills $\left|\left(\delta_{\alpha}\left(t^{j}, j\right), \bar{\delta}_{\beta}\left(t^{j}, j\right)\right)\right| \leq \bar{\delta}$ for all $j \geq J$ such that $t^{j}+j \geq \bar{s}_{1}$. Noting that $(J, \epsilon)$-PE implies $(j, \epsilon)$ PE for all $j \geq J$, we thus conclude that there exists a $J_{2} \geq \max \left\{J, J^{\prime}, \inf _{j \in \mathbb{N}} t^{j}+j \geq \bar{s}_{1}\right\}$ such that $\alpha^{\star}=\Pi_{\eta}(\theta) w$ is $\left(J_{2}, \epsilon^{\prime}\right)$-PE, and the map $\theta_{\alpha^{\star}, \beta^{\star}}^{\circ}(t, j)$ is a singleton for all $\left.(t, j) \in \operatorname{dom}(\mathbf{w}, \chi, z, \theta)\right|_{\geq \bar{s}_{2}}$, having denoted $\bar{s}_{2}:=t_{J_{2}}+J_{2}$.

2) Stability: In view of the aforementioned bounds $\Delta_{1}$ and $\Delta_{2}$ on $(\alpha, \beta)$ and $\left(\alpha^{\star}, \beta^{\star}\right)$, Proposition 1 implies that, with the same $\rho>0$, for each solution pair $((\mathbf{w}, \chi, z, \theta), \nu)$ to $(23)$ there exist $z^{\star}: \operatorname{dom}(\mathbf{w}, \chi, z, \theta) \rightarrow$ $\mathcal{Z}$ such that, for all $(t, j) \in \Gamma\left(\left.\operatorname{dom}(\mathbf{w}, \chi, z, \theta)\right|_{\geq \bar{s}_{2}}\right)$ and with $\tilde{z}:=z-z^{\star}$, we have

$$
\left|\tilde{z}^{+}\right|^{2} \leq \mu^{2}|\tilde{z}|^{2}+\rho r_{3} V(w, \chi, z, \theta)+\rho r_{4}|\nu|^{2},
$$

where we omitted the argument $(t, j)$ and we let $\tilde{z}^{+}:=$ $\tilde{z}(t, j+1)$. In the following, for an arbitrary $\epsilon>0$, we let $\mathcal{S}_{\epsilon}$ be the class of the solution pairs $((\mathbf{w}, \chi, z, \theta), \nu)$ to the closed-loop system with $\mathrm{T} \geq \mathrm{T}_{\epsilon}^{\star}$ and such that $\alpha$ is $(J, \epsilon)$-PE for some $J \in \mathbb{N}$. We stress that the above discussion, and thus in particular, that $\alpha^{\star}=\Pi_{\eta}(\theta) w$ is $\left(J_{2}, \epsilon^{\prime}\right)$-PE and $\theta_{\alpha^{\star}, \beta^{\star}}^{\circ}(t, j)$ is a singleton for $t+j \geq \bar{s}_{2}$, holds for all such solutions, with only $J_{2}$ and $\bar{s}_{2}$ that possibly depend on the particular solution. In the following we will make reference to the solution-dependent quantities introduced above ( such as $J_{2}$ and $\bar{s}_{2}$ ) with the remark that they are meant to be defined in the same way as before and they refer to the particular solution considered.

\section{3) $(J, \epsilon)-P E$ and $A 4$ yield the internal model property}

We show now that the single-valued solution to the identification problem associated to the quasi steady-state inputs is independent on $\theta$ and coincides with $\omega$ of (24). In view of $\mathrm{A} 4$, there exists $\omega \in \mathcal{E}$ such that (24) holds. As a consequence, (29) yields

$$
\begin{aligned}
\Pi_{\eta_{d}}(\theta) S & =\Pi_{\eta_{1}}(\theta) S^{d} \\
& =\Pi_{\eta_{1}}(\theta)\left(\omega_{d} S^{d-1}+\cdots+\omega_{2} S+\omega_{1} I\right)
\end{aligned}
$$

Then, for any solution pair $((\mathbf{w}, \chi, z), \nu)$ to the closedloop system, (29) and (32) yield

$$
\begin{aligned}
& \left|\beta^{\star}\left(t^{j}, j\right)-\left(\omega^{T} \otimes I_{n_{e}}\right) \alpha^{\star}\left(t^{j}, j\right)\right| \\
& =\mid\left(\Pi_{\eta_{d}}\left(\theta\left(t^{j}, j\right)\right) S\right. \\
& \left.\quad-\Pi_{\eta_{1}}\left(\theta\left(t^{j}, j\right)\right)\left(\omega_{d} S^{d-1}+\cdots+\omega_{1} I\right)\right) w\left(t^{j}, j\right) \mid=0
\end{aligned}
$$

for all $\left(t^{j}, j\right) \in \operatorname{dom}(\mathbf{w}, \chi, z, \theta)$. By definition of $\mathcal{J}_{\alpha^{\star}, \beta^{\star}}$ in (11), this also implies that $\mathcal{J}_{\alpha^{\star}, \beta^{\star}}(t, j)(\omega)=0$, and thus that $\omega \in \theta_{\alpha^{\star}, \beta^{\star}}^{\circ}(t, j)$, for all $(t, j) \in \operatorname{dom}(\mathbf{w}, \chi, z, \theta)$. Pick $((\mathbf{w}, \chi, z, \theta), \nu) \in \mathcal{S}_{\epsilon}$, as $\alpha^{\star}$ is $\left(J_{2}, \epsilon^{\prime}\right)-\mathrm{PE}$ and $\theta_{\alpha^{\star}, \beta^{\star}}^{\circ}(t, j)$ is single valued for all $\left.(t, j) \in \operatorname{dom}(\mathbf{w}, \chi, z, \theta)\right|_{\geq \bar{s}_{2}}$, then necessarily

$$
\theta_{\alpha^{\star}, \beta^{\star}}^{\circ}(t, j)=\{\omega\} \subset \mathcal{E},\left.\quad \forall(t, j) \in \operatorname{dom}(\mathbf{w}, \chi, z, \theta)\right|_{\geq \bar{s}_{2}} .
$$

A further consequence of (29) is that

$$
\begin{aligned}
\Pi_{e}(\omega) & =\Pi_{\eta_{d}}(\omega) S-\left(\omega^{T} \otimes I_{n_{e}}\right) \Pi_{\eta}(\omega) \\
& =\Pi_{\eta_{1}}(\omega)\left(S^{d}-\omega_{d} S^{d-1}-\cdots-\omega_{1} I\right)=0
\end{aligned}
$$

so as if limsup $|\chi-\Pi(\omega) w|$ is proportional to limsup $|\nu|$, then (25) is proved. In the next paragraph we show that this is the case whenever $T$ is sufficiently large.

\section{4) Large $T$ yields small gain}

Finally, we show here that if the jump times are distant enough, a small-gain like condition holds, and "modulo $\nu$ ", $\theta$ tends to the optimum $\omega$ and $\chi$ to the error-zeroing steady state $\Pi(\omega) w$. Thus the claim of the theorem follows. Pick $((\mathbf{w}, \chi, z, \theta), \nu) \in \mathcal{S}_{\epsilon}$. As $\omega \in \mathcal{E}$, in view of (33) and of Proposition 1, for all $\left.(t, j) \in \operatorname{dom}(\mathbf{w}, \chi, z, \theta)\right|_{\geq \bar{s}_{2}}$ we have $\omega=\theta^{\star}(t, j)$, with $\theta^{\star}(t, j)$ the unique element of $p_{\mathcal{E}}\left(R^{\star}(t, j)^{\dagger} v^{\star}(t, j)\right)$. Then Lemma 4 can be invoked to claim the existence of $J_{3} \geq J_{2}$ and $r_{5} \geq 0$, depending on $\epsilon$, such that, with $\bar{s}_{3}:=t_{J_{3}}+J_{3}$, the following holds

$$
|\theta(t, j)-\omega|^{2} \leq r_{5}|\tilde{z}(t, j)|^{2}
$$

for all $\left.(t, j) \in \operatorname{dom}(\mathbf{w}, \chi, z, \theta)\right|_{\geq \bar{s}_{3}}$. As a consequence of (35) and $(31)$, for all $(t, j) \in \Gamma\left(\left.\operatorname{dom}(\mathbf{w}, \chi, z, \theta)\right|_{\geq \bar{s}_{3}}\right)$, we obtain

$$
\begin{aligned}
& \left|\theta-\theta^{+}\right|^{2}=\left|\theta-\omega+\omega-\theta^{+}\right|^{2} \leq|\theta-\omega|^{2}+\left|\theta^{+}-\omega\right|^{2} \\
& \leq r_{5}\left(|\tilde{z}|^{2}+\left|\tilde{z}^{+}\right|^{2}\right) \leq r_{6}|\tilde{z}|^{2}+r_{7} V(w, \chi, z, \theta)+r_{8}|\nu|^{2}
\end{aligned}
$$

with $r_{6}:=r_{5}\left(1+\mu^{2}\right), r_{7}:=r_{5} \rho r_{3}, r_{8}:=r_{5} \rho r_{4}$ and where again we omitted the argument $(t, j)$ and we let $\theta^{+}:=\theta(t, j+1)$ and $\tilde{z}^{+}:=\tilde{z}(t, j+1)$. We further develop (28) to obtain, for all $(t, j) \in \Gamma\left(\left.\operatorname{dom}(\mathbf{w}, \chi, z, \theta)\right|_{\geq \bar{s}_{3}}\right)$

$V\left(w^{+}, \chi^{+}, z^{+}, \theta^{+}\right) \leq r_{9} V(w, \chi, z, \theta)+r_{10}|\tilde{z}|^{2}+r_{11}|\nu|^{2}$ 
being $r_{9}:=r_{1}+r_{2} r_{7}, r_{10}:=r_{2} r_{6}$ and $r_{11}:=r_{2} r_{8}$. In summary, for all $\left.(t, j) \in \operatorname{dom}(\mathbf{w}, \chi, z, \theta)\right|_{\geq \bar{s}_{3}}$ such that $t \in\left(t_{j}, t_{j+1}\right)$ we have:

$$
\dot{V}(w, \chi, z, \theta) \leq-\lambda V(w, \chi, z, \theta)+r_{0}|\nu|^{2}, \quad \frac{d|\tilde{z}|^{2}}{d t}=0
$$

and for all $(t, j) \in \Gamma\left(\left.\operatorname{dom}(\mathbf{w}, \chi, z)\right|_{\geq \bar{s}_{3}}\right)$

$$
\begin{aligned}
V\left(w^{+}, \chi^{+}, z^{+}, \theta^{+}\right) & \leq r_{9} V(w, \chi, z, \theta)+r_{10}|\tilde{z}|^{2}+r_{11}|\nu|^{2} \\
\left|\tilde{z}^{+}\right|^{2} & \leq \mu^{2}|\tilde{z}|^{2}+\rho r_{3} V(w, \chi, z, \theta)+\rho r_{4}|\nu|^{2},
\end{aligned}
$$

where we omitted the argument $(t, j)$ and we let $\left(w^{+}, \chi^{+}, z^{+}, \theta^{+}, \tilde{z}^{+}\right):=(w(t, j+1), \chi(t, j+1), z(t, j+$ 1), $\theta(t, j+1), \tilde{z}(t, j+1))$. We then have the following:

Lemma 5 There exist $c_{0} \geq 0$ and $\underline{\mathrm{T}}_{2}^{\star} \geq \underline{\mathrm{T}}_{\epsilon}^{\star}$, independent on $J_{3}$, such that for any solution pair in $\mathcal{S}_{\epsilon}$ with $\underline{\mathrm{T}} \geq \underline{\mathrm{T}}_{2}^{\star}$

$$
\limsup \left(V(w, \chi, z, \theta)+|\tilde{z}|^{2}\right) \leq c_{0} \limsup |\nu|^{2} .
$$

Lemma 5 implies in particular

$$
\begin{aligned}
& \limsup |\chi-\Pi(\theta) w| \leq c_{1} \limsup |\nu| \\
& \limsup |\theta-\omega| \leq c_{2} \limsup |\nu|
\end{aligned}
$$

for $c_{1}:=\sqrt{c_{0} / \underline{\sigma}}$ and $c_{2}:=\sqrt{r_{5} c_{0}}$. In view of $(34)$, $\Pi_{e}(\omega)=0$, so that

$$
\begin{aligned}
& |e|=\left|e-\Pi_{e}(\omega) w\right| \\
& \leq\left|C_{e}\right||\chi-\Pi(\omega)|+\left|C_{e}\right||w||\Pi(\theta)-\Pi(\omega)|+\left|N_{e}\right||\nu| .
\end{aligned}
$$

As $\Pi(\cdot)$ is Lipschitz on $\mathcal{E}$, this suffices to conclude (25), with $c:=\left|C_{e}\right| c_{1}+\left|C_{e}\right||W| L_{\Pi} c_{2}+\left|N_{e}\right|$, where $L_{\Pi}$ is the Lipschitz constant of $\Pi$ on $\mathcal{E}$. The result of the theorem follows then from the arbitrariness of $\epsilon$.

\section{A Numerical Example}

We consider here a plant of the form (2) with

$$
A=\left(\begin{array}{ccc}
1 & 1 & 1 \\
-1 & 0 & 1 \\
1 & 1 & 0
\end{array}\right), \quad B=\left(\begin{array}{ll}
0 & 0 \\
0 & 1 \\
1 & 2
\end{array}\right)
$$

The control goal is to drive $y_{1}:=x_{2}$ to a desired set point $y_{1}^{\star}$ chosen by the user and to make $y_{2}:=x_{3}$ follow a sinusoid $y_{2}^{\star}(t)$ at any desired frequency, despite the disturbances $P w(t)$ acting on the system. We suppose that $w(t)$ is a combination of a constant term, a harmonic at the same frequency of $y_{2}^{\star}(t)$ and a third unknown harmonic. The disturbance $P w(t)$ and the references $y_{1}^{\star}$ and
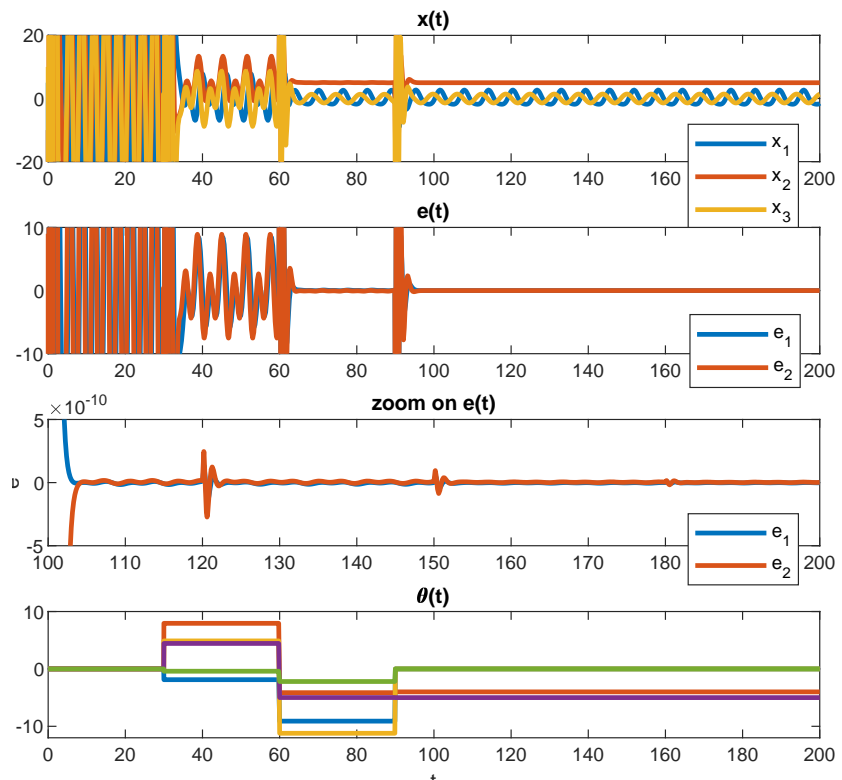

Figure 2. Simulation results: The first plot shows the time evolution of the state $x$, the second plot the regulation errors $e(t)$, the third plot is a zoom on the regulation errors and the last plot shows the evolution of the parameters $\theta$.

$y_{2}^{\star}(t)$ can be thus modeled as outputs of an exosystem of the form (3) with $S:=\operatorname{blkdiag}\left(S_{1}, S_{2}, S_{3}\right)$, where

$$
S_{1}=\gamma_{1}\left(\begin{array}{cc}
0 & 1 \\
-1 & 0
\end{array}\right), \quad S_{2}=\gamma_{2}\left(\begin{array}{cc}
0 & 1 \\
-1 & 0
\end{array}\right), \quad S_{3}=0
$$

and with $\gamma_{1}, \gamma_{2} \in \mathbb{R}_{>0}$ unknown parameters. We define the regulator errors as $e_{1}:=y_{1}-y_{1}^{\star}$ and $e_{2}:=y_{2}-y_{2}^{\star}$, that are obtained as in (4) with the choice

$$
C_{e}=\left(\begin{array}{ccc}
0 & 1 & 0 \\
0 & 0 & 1
\end{array}\right) \quad Q_{e}:=\left(\begin{array}{ccccc}
0 & 0 & 0 & 0 & -1 \\
-1 & 0 & 0 & 0 & 0
\end{array}\right)
$$

and noting that different set-points $y_{1}^{\star}$, as well as different amplitudes and phases for $y_{2}^{\star}(t)$ can be obtained by changing the initial conditions of $w$ and need not be known at the design stage. The amplitude and the phase of the disturbance $P w$ depends, in addition to depending on $w(0)$, depends on the matrix $P$ which is not known by the designer. It is worth noting that if we let $x^{*}:=\Pi w$ and $u^{*}:=\Gamma w$ be the corresponding steady state functions such that $e^{*}:=C_{e} x^{*}+Q_{e} w=0$, then, by letting $\tilde{x}:=x-x^{*}$, we obtain that $e=0$ and $u=u^{\star}$ imply $^{2}$

$$
\dot{\tilde{x}}_{1}=\tilde{x}_{1}
$$

\footnotetext{
2 This can be verified by noting that $\Pi$ and $\Gamma$ fulfill the regulator equations $\Pi S=A \Pi+B \Gamma+P$ and $0=C_{e} \Pi+Q_{e}$, and that $e=0$ implies $\tilde{x}_{2}=\tilde{x}_{3}=0$.
} 
so that the plant considered is not minimum phase relative to the ideal "steady state locus" given by the set graph $\Pi:=\left\{(w, x) \in \mathbb{R}^{5} \times \mathbb{R}^{3}: x=\Pi w\right\}$ and, as a consequence, this example does not fit in the frameworks addressed in the existing literature. In the simulation, for simplicity, we used a state-feedback stabilizer, i.e. we assumed $y=\operatorname{col}\left(e, x_{1}\right)$ and $u=K(\theta) y$ with $K(\theta)$ properly designed. Figure 2 shows the result of a simulation of the proposed control system implemented with $d=5, \mu=0.9$ and with $T=30 \mathrm{~s}$. In the simulation we let $y_{2}^{*}=5, \gamma_{1}=1, \gamma_{2}=2$, $w(0)=\operatorname{col}\left(1,-1,0,1, y_{2}^{*}\right), x(0)=\operatorname{col}(5,-10,10)$ and $P=\operatorname{col}\left(\left(\begin{array}{lllll}1 & 0 & 1 & 0 & 1\end{array}\right),\left(\begin{array}{lllll}1 & 0 & 1 & 0 & 1\end{array}\right),\left(\begin{array}{lllll}1 & 0 & 1 & 0 & 1\end{array}\right)\right)$.

\section{Conclusions and Future Work}

In this paper we proposed a control design to address a class of output regulation problems for linear systems in the presence of uncertainties in the exosystem. The proposed regulator combines a canonical continuous-time internal model and a discrete-time identifier that adapts the internal model parameters. We have shown that if the identifier is slow enough the regulator ensures boundedness of the closed-loop trajectories and, if an upper bound on the order of the exosystem is known, then along the solutions that satisfy a persistency of excitation condition asymptotic regulation is achieved. We also showed robustness of the proposed scheme to sufficiently small unmodeled disturbances. The main interest for future developments concerns the synergy between identification and control and the relation between the prediction performances of the identifier and the regulation performance of the control scheme. As in $[19,21]$, indeed, the asymptotic "optimality" of the identifier turned out here to be the key to obtain asymptotic regulation. Future researches will mainly focus on the extension of the control paradigm to more general plants modeled by nonlinear differential inclusions and the integration into the framework of more general identifiers, by eventually introducing stochastic identifiers.

\section{A Proofs}

\section{A.1 Proof of Lemma 1}

Let

$$
F(\lambda):=\left(\begin{array}{cc}
A-\lambda I & B \\
C_{e} & 0
\end{array}\right) .
$$

We first prove that the set $\mathcal{U}:=\{\lambda \in \mathbb{C}: \operatorname{rank} F(\lambda)<$ $\left.n+n_{e}\right\}$ is finite. Pick $\lambda \in \mathbb{C} / \sigma(A)$. Then $A-\lambda I$ is invertible and the matrix

$$
M(\lambda):=\left(\begin{array}{cc}
I_{n} & 0_{n \times n_{e}} \\
C_{e}(A-\lambda I)^{-1} & -I_{n_{e}}
\end{array}\right)
$$

is well-defined and full rank. Thus

$$
\begin{aligned}
\operatorname{rank} F(\lambda) & =\operatorname{rank}(M(\lambda) F(\lambda)) \\
& =\operatorname{rank}\left(\begin{array}{cc}
A-\lambda I & B \\
0_{n_{e}} & C_{e}(A-\lambda I)^{-1} B
\end{array}\right) \\
& \geq n+\operatorname{rank}\left(C_{e}(A-\lambda I)^{-1} B\right) .
\end{aligned}
$$

By assumption, as rank $B \geq n_{e}$, the number of $\lambda \in \mathbb{C}$ for which $\operatorname{rank}\left(C_{e}(A-\lambda I)^{-1} B\right)<n_{e}$ is finite. Since $\sigma(A)$ has at most $n$ elements, then we conclude that $\mathcal{U}$ is finite. Let $\mathcal{P}^{d}$ denote the set of monic polynomials of degree $d$ with coefficients in $\mathbb{R}$. Each element of $\mathcal{P}^{d}$ can be written as $p_{a}(s)=s^{d}+a_{d} s^{d-1}+\cdots+a_{2} s+a_{1}$, for some $a \in \mathbb{R}^{d}$, so as there is a natural isomorphism $\iota: \mathbb{R}^{d} \rightarrow \mathcal{P}^{d}$, $a=\operatorname{col}\left(a_{1}, \ldots, a_{d}\right) \mapsto p_{a}(s)$. Let $P(\lambda) \subset \mathcal{P}^{d}$ denote the set of polynomials in $\mathcal{P}^{d}$ that have $\lambda$ as a root. Suppose $\lambda \in \mathbb{R}$, each element in $P(\lambda)$ can be univocally written as

$$
\begin{aligned}
p_{a}(s) & =(s-\lambda)\left(s^{d-1}+a_{d-1} s^{d-2}+\cdots+a_{2} s+a_{1}\right) \\
& =s^{d}+\left(a_{d-1}-\lambda\right) s^{d-1}+\cdots+\left(a_{1}-a_{2} \lambda\right) s-a_{1} \lambda,
\end{aligned}
$$

so as $\iota^{-1}(P(\lambda))$ is the $(d-1)$-dimensional affine subspace of $\mathbb{R}^{d}$ given by $\iota^{-1}(P(\lambda))=\left\{\left(-\lambda a_{1}, a_{1}-\lambda a_{2}, \ldots, a_{d-2}-\right.\right.$ $\left.\left.\lambda a_{d-1}, a_{d-1}-\lambda\right) \in \mathbb{R}^{d}:\left(a_{1}, \ldots, a_{d-1}\right) \in \mathbb{R}^{d-1}\right\}$. If instead $\lambda \in \mathbb{C} / \mathbb{R}$, in the same way as before it can be seen that $\iota^{-1}(P(\lambda))$ is a $(d-2)$-dimensional affine subspace of $\mathbb{R}^{d}$. As $\mathcal{Q}$ can be written as $\mathcal{Q}=\left\{\theta \in \mathbb{R}^{d}: \iota(\theta) \in\right.$ $P(\lambda), \lambda \in \mathcal{U}\}=\cup_{\lambda \in \mathcal{U} \iota^{-1}}(P(\lambda))$, then $\mathcal{Q}$ is the union of a finite number of affine subspaces of $\mathbb{R}^{d}$ of dimension $d-1$ or $d-2$. Pick $r>0$ arbitrarily and notice that the set $\mathcal{Q}+\mathbb{B}_{r}$ is open as it is a union of open sets. Hence $\mathbb{R}^{d} \backslash\left(\mathcal{Q}+\mathbb{B}_{r}\right)$ is closed and it remains to show that it is not empty. We thus notice that for each $\lambda \in \mathcal{U}$ we can write $\iota^{-1}(P(\lambda))=x_{\lambda}+\operatorname{Im} A_{\lambda}$, for some $x_{\lambda} \in \mathbb{R}^{d}$ and $A_{\lambda} \in \mathbb{R}^{d \times(d-1)}$. Pick any $\lambda \in \mathcal{U}$ and define the set $S:=\left\{x_{\lambda}+y+v \in \mathbb{R}^{d-1}: y \in \operatorname{Im} A_{\lambda}, v \in\left(\operatorname{Im} A_{\lambda}\right)^{\perp}\right\}$. We now show that $S \cap\left(\mathbb{R}^{d} \backslash\left(\mathcal{Q}+\mathbb{B}_{r}\right)\right) \neq \emptyset$. For, let $\mathcal{U}_{\lambda} \subset \mathcal{U}$ be the set of $\mu \in \mathcal{U}$ such that $\operatorname{Im} A_{\lambda} \subseteq \operatorname{Im} A_{\mu}$. Then for each $\mu \in \mathcal{U}_{\lambda}$, each $y \in \operatorname{Im} A_{\lambda}$, and with $p=$ $x_{\lambda}+y+v \in S$, in view of [31, Thm. 4.9], we have

$$
\begin{aligned}
|p|_{\iota^{-1}(P(\mu))} & =\inf _{z \in \operatorname{Im} A_{\mu}}\left|x_{\mu}+z-x_{\lambda}-y-v\right| \\
& =\left|\left(x_{\mu}-x_{\lambda}\right)^{\prime}-v^{\prime}\right|
\end{aligned}
$$

where $\left(x_{\mu}-x_{\lambda}\right)^{\prime}$ and $v^{\prime}$ denote the projection of $x_{\mu}-x_{\lambda}$ and $v$ onto $\left(\operatorname{Im} A_{\mu}\right)^{\perp}$. Hence, for each $v \in\left(\operatorname{Im} A_{\lambda}\right)^{\perp}$ fulfilling $\left|v^{\prime}\right|>r+\max _{\mu \in \mathcal{U}_{\lambda}}\left|x_{\mu}-x_{\lambda}\right|$ we obtain $|p|_{\iota^{-1}(P(\mu))} \geq\left|v^{\prime}\right|-\left|\left(x_{\mu}-x_{\lambda}\right)^{\prime}\right|>r$, and this in turn shows that, for every $y \in \operatorname{Im} A_{\lambda}$ and for sufficiently large $v, p=x_{\lambda}+y+v \in \mathbb{R}^{d} \backslash\left(\cup_{\mu \in \mathcal{U}_{\lambda}} \iota^{-1}(P(\mu))+\mathbb{B}_{r}\right)$. Pick now $\mu \in \mathcal{U} \backslash \mathcal{U}_{\lambda}$ and fix a $v \in\left(\operatorname{Im} A_{\lambda}\right)^{\perp}$ satisfying the above bound, then $\operatorname{Im} A_{\lambda} \cap\left(\operatorname{Im} A_{\mu}\right)^{\perp} \neq \emptyset$ and we get

$$
\begin{aligned}
|p|_{\iota^{-1}(P(\mu))} & =\inf _{z \in \operatorname{Im} A_{\mu}}\left|x_{\mu}+z-x_{\lambda}-y-v\right| \\
& =\left|\left(x_{\mu}-x_{\lambda}\right)^{\prime \prime}-y^{\prime \prime}-v^{\prime \prime}\right|
\end{aligned}
$$


with $\left(x_{\mu}-x_{\lambda}\right)^{\prime \prime}, y^{\prime \prime}$ and $v^{\prime \prime}$ the projections of $x_{\mu}-x_{\lambda}$, $y$ and $v$ onto $\left(\operatorname{Im} A_{\mu}\right)^{\perp}$. Hence choosing $y$ so that $\left|y^{\prime \prime}\right|>$ $r+\max _{\mu \in \mathcal{U} \backslash \mathcal{U}_{\lambda}}\left|x_{\mu}-x_{\lambda}\right|+\left|v^{\prime \prime}\right|$ yields $|p|_{\iota^{-1}(P(\mu))}>r$, and this in turn proves that there exists a $p \in S$ satisfying $p \in \mathbb{R}^{d} \backslash\left(\mathcal{Q}+\mathbb{B}_{r}\right)$. Hence the claim.

\section{A.2 Proof of Proposition 1}

Recall that, for $z=(R, v) \in \mathcal{Z},|z|^{2}=|R|^{2}+|v|^{2}$. Thus

$$
\begin{aligned}
& \left|z_{1}^{+}-z_{2}^{+}\right|^{2} \\
& =\left|\mu\left(R_{1}-R_{2}\right)+\gamma\left(\alpha_{1}\right) \gamma\left(\alpha_{1}\right)^{T}-\gamma\left(\alpha_{2}\right) \gamma\left(\alpha_{2}\right)^{T}\right|^{2} \\
& \quad+\left|\mu\left(v_{1}-v_{2}\right)+\gamma\left(\alpha_{1}\right) \beta_{1}-\gamma\left(\alpha_{2}\right) \beta_{2}\right|^{2} \\
& \leq \mu^{2}\left|z_{1}-z_{2}\right|^{2}+\left|\gamma\left(\alpha_{1}\right) \gamma\left(\alpha_{1}\right)^{T}-\gamma\left(\alpha_{2}\right) \gamma\left(\alpha_{2}\right)^{T}\right|^{2} \\
& \quad+\left|\gamma\left(\alpha_{1}\right) \beta_{1}-\gamma\left(\alpha_{2}\right) \beta_{2}\right|^{2},
\end{aligned}
$$

and the first point follows from the fact that $\mid \gamma\left(\alpha_{1}\right) \gamma\left(\alpha_{1}\right)^{T}$ $\left.\gamma\left(\alpha_{2}\right) \gamma\left(\alpha_{2}\right)^{T}\right|^{2}=\mid \gamma\left(\alpha_{1}\right)\left(\gamma\left(\alpha_{1}\right)-\gamma\left(\alpha_{2}\right)\right)^{T}-\left(\gamma\left(\alpha_{2}\right)-\right.$ $\left.\gamma\left(\alpha_{1}\right)\right)\left.\gamma\left(\alpha_{2}\right)^{T}\right|^{2} \leq\left(\left|\alpha_{1}\right|^{2}+\left|\alpha_{2}\right|^{2}\right)\left|\alpha_{1}-\alpha_{2}\right|^{2}$ and $\left|\gamma\left(\alpha_{1}\right) \beta_{1}-\gamma\left(\alpha_{2}\right) \bar{\beta}_{2}\right|^{2}=\mid \gamma\left(\alpha_{1}\right)\left(\beta_{1}-\beta_{2}\right)-\left(\gamma\left(\alpha_{2}\right)-\right.$ $\left.\gamma\left(\alpha_{1}\right)\right)\left.\beta_{2}\right|^{2} \leq\left|\alpha_{1}\right|^{2}\left|\beta_{1}-\beta_{2}\right|^{2}+\left|\beta_{2}\right|^{2}\left|\alpha_{1}-\alpha_{2}\right|^{2}$, with $\rho=2\left|\alpha_{1}\right|^{2}+\left|\alpha_{2}\right|^{2}+\left|\beta_{2}\right|^{2}$. For what concerns the second point, pick $(t, j) \in \operatorname{dom}\left(\alpha^{\star}, \beta^{\star}\right)$. Then $\theta_{\alpha^{\star}, \beta^{\star}}^{\circ}(t, j)$ is the set of points that annihilate the gradient of $\mathcal{J}_{\alpha^{\star}, \beta^{\star}}(\theta)(t, j)$ with respect to $\theta$. Simple computations show that such a set is given by (13)-(14), with $\left(\alpha^{\star}, \beta^{\star}\right)$ in place of $(\alpha, \beta)$. By direct solution it is possible to show that $z^{\star}:=\left(R^{\star}, v^{\star}\right)$, with $R^{\star}$ and $v^{\star}$ given by $(14)$ is exactly the solution to the subsystem $z$ of $(9)$ with $(\alpha, \beta)=\left(\alpha^{\star}, \beta^{\star}\right)$ originating from $z^{\star}(0,0)=\left(\gamma\left(\alpha^{\star}(0,0)\right) \gamma\left(\alpha^{\star}(0,0)\right)^{T}, \gamma\left(\alpha^{\star}(0,0)\right) \beta^{\star}(0,0)\right)$, and this concludes the proof.

\section{A.3 Proof of Lemma 3}

Let $\ell_{\infty}$ be the space of bounded sequences $s=\left(s_{n}\right)_{n \in \mathbb{N}}$ and, for $s \in \ell_{\infty}$, let $|s|_{n_{1}, n_{2}}:=\sup _{n_{1} \leq n \leq n_{2}}\left|s_{n}\right|$. Let $\phi(\alpha):=\operatorname{col}\left(\alpha\left(t^{0}, 0\right), \alpha\left(t^{1}, 1\right), \ldots\right) \in \ell_{\infty}$ and, for $k, j \in$ $\mathbb{N}$, let $\Sigma_{k}^{j}: \ell_{\infty} \rightarrow \mathbb{R}^{d \times d}$ be the function

$$
s \mapsto \Sigma_{k}^{j}(s):=\sum_{i=k}^{j-1} \mu^{j-i-1} \gamma\left(s_{i}\right) \gamma\left(s_{i}\right)^{T} .
$$

For any two $q_{1}, q_{2} \in \mathbb{R}^{n_{e} d}$ there exists $c_{0}>0$ such that $\left|\gamma\left(q_{1}\right) \gamma\left(q_{1}\right)^{T}-\gamma\left(q_{2}\right) \gamma\left(q_{2}\right)^{T}\right| \leq c_{0}\left|q_{1}-q_{2}\right|$. As a consequence, for each two $s^{1}, s^{2} \in \ell_{\infty}$ there exists $c_{0}>0$ such that, for each $k, j \in \mathbb{N}$, we have

$$
\begin{aligned}
& \left|\Sigma_{k}^{j}\left(s_{1}\right)-\Sigma_{k}^{j}\left(s_{2}\right)\right| \\
& \quad=\sum_{i=k}^{j-1} \mu^{j-i-1}\left(\gamma\left(s_{i}^{1}\right) \gamma\left(s_{i}^{1}\right)^{T}-\gamma\left(s_{i}^{2}\right) \gamma\left(s_{i}^{2}\right)^{T}\right) \\
& \quad \leq\left(\sum_{i=k}^{j-1} \mu^{j-i-1}\right) c_{0}\left|s^{1}-s^{2}\right|_{k, \infty} .
\end{aligned}
$$

Noting that

$$
\sum_{i=k}^{j-1} \mu^{j-i-1} \leq \sum_{i=0}^{j-1} \mu^{j-i-1}=\sum_{\ell=0}^{j-1} \mu^{\ell} \leq \sum_{\ell=0}^{\infty} \mu^{\ell}=\frac{1}{1-\mu}
$$

we thus obtain

$$
\left|\Sigma_{k}^{j}\left(s_{1}\right)-\Sigma_{k}^{j}\left(s_{2}\right)\right| \leq c_{1}\left|s^{1}-s^{2}\right|_{k, \infty},
$$

for each $k, j \in \mathbb{N}$ and with $c_{1}:=c_{0} /(1-\mu)$. As the map $R \in \mathbb{R}^{n_{e} d \times n_{e} d} \mapsto \min \sigma(R) \in \mathbb{R}_{\geq 0}$ is continuous, for every $v>0$ there exists $r_{v}>0$ such that

$$
\begin{aligned}
& \left|\Sigma_{0}^{j}(\phi(\alpha))-\Sigma_{0}^{j}\left(\phi\left(\alpha^{\star}\right)\right)\right| \leq r_{v} \\
& \quad \Longrightarrow\left|\min \sigma\left(\Sigma_{0}^{j}(\phi(\alpha))\right)-\min \sigma\left(\Sigma_{0}^{j}\left(\phi\left(\alpha^{\star}\right)\right)\right)\right| \leq v .
\end{aligned}
$$

As $\Sigma_{0}^{j}$ has symmetric positive semi-definite values then

$$
\begin{aligned}
& \min \sigma\left(\Sigma_{0}^{j}\left(\phi\left(\alpha^{\star}\right)\right)\right)=\left|\min \sigma\left(\Sigma_{0}^{j}\left(\phi\left(\alpha^{\star}\right)\right)\right)\right| \\
& =\mid \min \sigma\left(\Sigma_{0}^{j}(\phi(\alpha))\right) \\
& \quad-\left(\min \sigma\left(\Sigma_{0}^{j}(\phi(\alpha))\right)-\min \sigma\left(\Sigma_{0}^{j}\left(\phi\left(\alpha^{\star}\right)\right)\right)\right) \mid \\
& \geq|| \min \sigma\left(\Sigma_{0}^{j}(\phi(\alpha))\right) \mid \\
& \quad-\left|\min \sigma\left(\Sigma_{0}^{j}(\phi(\alpha))\right)-\min \sigma\left(\Sigma_{0}^{j}\left(\phi\left(\alpha^{\star}\right)\right)\right)\right| \mid .
\end{aligned}
$$

Noting that $\Sigma_{0}^{j}(\phi(\alpha))$ is exactly the matrix appearing in (15), and since $\alpha$ is $(J, \epsilon)-\mathrm{PE}$, then $\min \sigma\left(\Sigma_{0}^{j}(\phi(\alpha))\right)>\epsilon$. Pick $\epsilon^{\prime}<\epsilon$ and $v \in\left(0, \epsilon-\epsilon^{\prime}\right)$ arbitrarily. Thus if for some $J^{\prime} \geq J$ we have $\left|\Sigma_{0}^{j}(\phi(\alpha))-\Sigma_{0}^{j}\left(\phi\left(\alpha^{\star}\right)\right)\right| \leq r_{v}$ for all $j \geq J^{\prime}$, then (A.2) and (A.3) give

$$
\min \sigma\left(\Sigma_{0}^{j}\left(\phi\left(\alpha^{\star}\right)\right)\right) \geq \epsilon-v \geq \epsilon^{\prime}
$$

for all $j \geq J^{\prime}$, that is the first claim. We thus have to show that (A.2) holds for sufficiently small $\bar{\delta}$. Note that, for each $j>J$

$$
\begin{aligned}
\left|\Sigma_{0}^{j}(\phi(\alpha))-\Sigma_{0}^{j}\left(\phi\left(\alpha^{\star}\right)\right)\right| \leq & \mu^{j-J}\left|\Sigma_{0}^{J}(\phi(\alpha))-\Sigma_{0}^{J}\left(\phi\left(\alpha^{\star}\right)\right)\right| \\
& +\left|\Sigma_{J}^{j}(\phi(\alpha))-\Sigma_{J}^{j}\left(\phi\left(\alpha^{\star}\right)\right)\right| .
\end{aligned}
$$

As the first term of (A.4) is a constant multiplied by $\mu^{j}$, and $\mu<1$, we claim the existence of $J^{\prime} \geq J$ such that in (A.4) we have that $\mu^{j-J}\left|\Sigma_{0}^{J}(\phi(\alpha))-\Sigma_{0}^{J}\left(\phi\left(\alpha^{\star}\right)\right)\right| \leq$ $r_{v} / 2$ for all $j \geq J^{\prime}$. Moreover, in view of (A.1), $\left|\phi\left(\delta_{\alpha}\right)\right|_{J, \infty}=\left|\phi(\alpha)-\phi\left(\alpha^{\star}\right)\right|_{J, \infty} \leq r_{v} /\left(2 c_{1}\right)$ implies $\left|\Sigma_{J}^{j}(\phi(\alpha))-\Sigma_{J}^{j}\left(\phi\left(\alpha^{\star}\right)\right)\right| \leq r_{v} / 2$ for all $j \geq J$. Thus (A.4) yields (A.2) for all $j \geq J^{\prime}$. This in turn proves the first claim, with $\bar{\delta}:=r_{v} /\left(2 c_{1}\right)$. To see that $\theta_{\alpha^{\star}, \beta^{\star}}^{\circ}(t, j)$ is single valued, notice that it is the set of $\theta$ in which the gradient of (11) vanishes, which is given by (13)(14) with $\left(\alpha^{\star}, \beta^{\star}\right)$ in place of $(\alpha, \beta)$. In view of $(14)$, $v^{\star}(t, j) \in \operatorname{Im} R^{\star}(t, j)$. Thus, noting that for $j \geq J^{\prime}$, $R^{\star}(t, j)=\Sigma_{0}^{j}\left(\phi\left(\alpha^{\star}\right)\right)$ is nonsingular, then (13) is a singleton and the second claim follows. 


\section{A.4 Proof of Lemma 4:}

For sake of readability, we will omit the time dependency. By letting $\theta_{i}^{u}(t, j):=R_{i}(t, j)^{\dagger} v_{i}(t, j)$, then for suitable selections $s_{\mathcal{E}}^{1}$ and $s_{\mathcal{E}}^{2}$ of $p_{\mathcal{E}}$, we obtain

$$
\begin{aligned}
\left|\theta_{1}-\theta_{2}\right|^{2} & =\left|s_{\mathcal{E}}^{1}\left(\theta_{1}^{u}\right)-s_{\mathcal{E}}^{2}\left(\theta_{2}^{u}\right)\right|^{2} \\
& =\left|s_{\mathcal{E}}^{1}\left(\theta_{1}^{u}\right)-\theta_{1}^{u}+\theta_{1}^{u}-\theta_{2}^{u}+\theta_{2}^{u}-s_{\mathcal{E}}^{2}\left(\theta_{2}^{u}\right)\right|^{2} \\
& \leq \inf _{\theta_{\mathcal{E}} \in \mathcal{E}}\left|\theta_{1}^{u}-\theta_{\mathcal{E}}\right|^{2}+\inf _{\theta_{\mathcal{E}} \in \mathcal{E}}\left|\theta_{2}^{u}-\theta_{\mathcal{E}}\right|^{2}+\left|\theta_{1}^{u}-\theta_{2}^{u}\right|^{2} \\
& \leq 3\left|\theta_{1}^{u}-\theta_{2}^{u}\right|^{2}=3\left|R_{1}^{\dagger} v_{1}-R_{2}^{\dagger} v_{2}\right|^{2} \\
& \leq 3\left|R_{1}^{\dagger}-R_{2}^{\dagger}\right|^{2}\left|v_{1}\right|^{2}+3\left|R_{2}^{\dagger}\right|^{2}\left|v_{1}-v_{2}\right|^{2} .
\end{aligned}
$$

In view of [32, Thm. 10.4.5], we have $\left|R_{1}^{\dagger}-R_{2}^{\dagger}\right|^{2} \leq$ $9 \max \left\{\left|R_{1}^{\dagger}\right|^{4},\left|R_{2}^{\dagger}\right|^{4}\right\}\left|R_{1}-R_{2}\right|^{2}$, which yields

$$
\begin{aligned}
& \left|\theta_{1}-\theta_{2}\right|^{2} \\
& \quad \leq 3 \max \left\{9 \max \left\{\left|R_{1}^{\dagger}\right|,\left|R_{2}^{\dagger}\right|\right\}^{4}\left|v_{1}\right|^{2},\left|R_{2}^{\dagger}\right|^{2}\right\}\left|z_{1}-z_{2}\right|^{2}
\end{aligned}
$$

By direct solution we obtain, for $i=1,2$

$$
\begin{aligned}
R_{i}(t, j)= & \mu^{j} R_{i}(0,0) \\
& +\sum_{k=0}^{j-1} \mu^{j-1-k} \gamma\left(\alpha_{i}\left(t^{k}, k\right)\right) \gamma\left(\alpha_{i}\left(t^{k}, k\right)\right)^{T}
\end{aligned}
$$

Since $\mu<1$ the first term of (A.6) vanishes exponentially with $j$. Thus using the fact that $\alpha_{i}$ is $\left(J_{i}, \epsilon_{i}\right)$-PE, the same arguments of Lemma 3 can be used to show that (A.6) implies that for any $\epsilon_{i}^{\prime} \in\left(0, \epsilon_{i}\right)$, there exists $J_{i}^{\prime} \geq J_{i}$ such that, for all $(t, j) \in \operatorname{dom} R_{i}$ such that $j \geq J_{i}^{\prime}, \min \sigma\left(R_{i}(t, j)\right) \geq \epsilon_{i}^{\prime}$. As a consequence, by letting $J:=\max \left\{J_{1}^{\prime}, J_{2}^{\prime}\right\}$ and $\epsilon:=\min \left\{\epsilon_{1}^{\prime}, \epsilon_{2}^{\prime}\right\}$, we obtain $\left|R_{i}(t, j)^{\dagger}\right| \leq 1 / \epsilon$ for all $(t, j) \in \operatorname{dom} R$ such that $j \geq J$. Thus the result follows from (A.5) by noting that boundedness of $\left(\alpha_{i}, \beta_{i}\right)$ for $i=1,2$ implies those of $v_{i}$.

\section{Proof of Lemma 5:}

Pick any $k \in(0, a)$ and $q \in\left(\mu^{2}, 1\right)$, and let

$$
\ell_{1} \in\left(0, q-\mu^{2}\right), \quad \ell_{2} \in\left(1, q /\left(\mu^{2}+\ell_{1}\right)\right) .
$$

then $0<\ell_{1}<1, \ell_{2}>1$ and $\left(\ell_{1}+\mu^{2}\right) \ell_{2}<q<1$. Let

$$
\psi \geq r_{10} / \ell_{1}, \quad \underline{\mathrm{T}}_{2}^{\star}:=\max \left\{\underline{\mathrm{T}}_{\epsilon}^{\star}, \frac{1}{k} \log \left(\frac{r_{9}+\rho r_{3} \psi}{q}\right)\right\}
$$

pick in the jump set of (23) $\overline{\mathrm{T}} \geq \underline{\mathrm{T}} \geq \underline{\mathrm{T}}_{2}^{\star}$ and let

$$
h \in\left(0, \log \left(\ell_{2}\right) / \overline{\mathrm{T}}\right) .
$$

Define the function

$$
W(\mathbf{w}, \chi, z, \theta, \tilde{z}):=e^{k \tau} V(w, \chi, z, \theta)+\psi e^{-h \tau}|\tilde{z}|^{2} .
$$

Then, clearly,

$$
V(w, \chi, z, \theta)+|\tilde{z}|^{2} \leq \max \left\{1, e^{h \overline{\mathrm{T}}} / \psi\right\} W(\mathbf{w}, \chi, z, \theta, \tilde{z}) .
$$

Pick a solution pair $((\mathbf{w}, \chi, z, \theta), \nu) \in \mathcal{S}_{\epsilon}$ with $\overline{\mathrm{T}} \geq \underline{\mathrm{T}} \geq$ $\underline{\mathrm{T}}_{2}^{\star}$. Then, using (18), for all $\left.(t, j) \in \operatorname{dom}(\mathbf{w}, \chi, z, \theta)\right|_{>\bar{s}_{3}}$ such that $t \in\left(t_{j}, t_{j+1}\right)$, (36) yields (we omit the time dependency)

$$
\dot{W}(\mathbf{w}, \chi, z, \theta, \tilde{z}) \leq-a_{W} W(\mathbf{w}, \chi, z, \theta, \tilde{z})+r_{12}|\nu|^{2},
$$

with $a_{W}:=\min \{a-k, h\}$ and $r_{12}:=e^{k \overline{\mathrm{T}}} r_{0}$. As $\tau^{+}=0$, for all $(t, j) \in \Gamma\left(\left.\operatorname{dom}(\mathbf{w}, \chi, z, \theta)\right|_{\geq \bar{s}_{3}}\right)$, instead, (37) yields

$$
\begin{aligned}
W\left(\mathbf{w}^{+}, \chi^{+}, z^{+}, \theta^{+}, \tilde{z}^{+}\right) \leq & \left(r_{9}+\rho r_{3}\right) V(w, \chi, z, \theta) \\
& +\left(r_{10}+\psi \mu^{2}\right)|\tilde{z}|^{2}+r_{13}|\nu|^{2},
\end{aligned}
$$

with $r_{13}:=r_{11}+\rho r_{4} \psi$. As for each $(t, j) \in \Gamma(\operatorname{dom}(\mathbf{w}, \chi$, $z, \theta)$ ), necessarily, $\underline{\mathrm{T}} \leq \tau(t, j) \leq \overline{\mathrm{T}}$, we get

$$
\begin{aligned}
& W\left(\mathbf{w}^{+}, \chi^{+}, z^{+}, \theta^{+}, \tilde{z}^{+}\right) \\
& \leq\left(r_{9}+\rho r_{3} \psi\right) e^{-k \underline{\mathrm{T}}} e^{k \tau} V(w, \chi, z, \theta) \\
&+\left(r_{10}+\psi \mu^{2}\right) e^{h \overline{\mathrm{T}}} e^{-h \tau}|\tilde{z}|^{2}+r_{13}|\nu|^{2} .
\end{aligned}
$$

(A.7)-(A.8) and $\underline{\mathrm{T}} \geq \underline{\mathrm{T}}_{2}^{\star}$ gives:

$$
\begin{aligned}
\left(r_{9}+\rho r_{3} \psi\right) e^{-k \underline{\mathrm{T}}} & \leq\left(r_{9}+\rho r_{3} \psi\right) e^{-k \underline{\mathrm{T}}_{2}^{*}} \leq q \\
\left(r_{10}+\psi \mu^{2}\right) e^{h \overline{\mathrm{T}}} & \leq \psi\left(r_{10} / \psi+\mu^{2}\right) \ell_{2} c \leq \psi q,
\end{aligned}
$$

so that we obtain

$$
W\left(\mathbf{w}^{+}, \chi^{+}, z^{+}, \theta^{+}, \tilde{z}^{+}\right) \leq q W(\mathbf{w}, \chi, z, \theta, \tilde{z})+r_{13}|\nu|^{2},
$$

and the claim follows from (A.9), (A.10) and (A.11).

\section{References}

[1] B. A. Francis and W. M. Wonham, "The internal model principle for multivariable regulators," Applied Mathematics \& Optimization, vol. 2, no. 2, pp. 170-194, 1975.

[2] B. A. Francis and W. M. Wonham, "The internal model principle of control theory," Automatica, vol. 12, pp. 457465, 1976.

[3] E. J. Davison, "The robust control of a servomechanism problem for linear time-invariant multivariable systems," IEEE Trans. Autom. Control, vol. AC-21, no. 1, pp. 25-34, 1976.

[4] F. Califano, M. Bin, A. Macchelli, and C. Melchiorri, "Stability analysis of nonlinear repetitive control schemes," IEEE Control Systems Letters, vol. 2, no. 4, pp. 773-778, 2018.

[5] M. Bin, D. Astolfi, L. Marconi, and L. Praly, "About robustness of internal model-based control for linear and nonlinear systems," in 2018 IEEE 58th Conf. Decision and Control (CDC), (Miami Beach, FL, USA), 2018. 
[6] R. Marino and P. Tomei, "Output regulation for linear systems via adaptive internal model," IEEE Trans. autom. control, vol. 40, no. 12, pp. 2199-2202, 2003.

[7] R. Marino and G. L. Santosuosso, "Regulation of linear systems with unknown exosystems of uncertain order," IEEE Trans. Autom. Control, vol. 52, no. 2, pp. 352-359, 2007.

[8] I. Muzimoto and Z. Iwai, "Adaptive output regulation of unknown MIMO linear systems with unknown exosystems," in Proc. European Control Conference 2007, (Kos, Greece), 2007.

[9] M. Bado and A. Ichikawa, "Adaptive output regulation for linear systems," in SICE-ICASE Int. Joint Conf. 2006, (Bexco, Busan, Korea), 2006.

[10] M. Bodson and S. C. Douglas, "Adaptive algorithms for the rejection of sinusoidal disturbances with unknown frequency," Automatica, vol. 33, no. 12, pp. 2213-2221, 1997.

[11] X. Guo and M. Bodson, "Analysis and implementation of an adaptive algorithm for the rejection of multiple sinusoidal disturbances," IEEE Trans. Control System Technology, vol. 17, no. 1, pp. 40-50, 2009.

[12] V. O. Nikiforov, "Adaptive non-linear tracking with complete compensation of unknown disturbances," European Journal of Control, vol. 4, pp. 132-139, 1998.

[13] Z. Ding, "Global stabilization and disturbance suppression of a class of nonlinear systems with uncertain internal model," Automatica, vol. 39, pp. 471-479, 2003.

[14] A. Serrani, A. Isidori, and L. Marconi, "Semiglobal nonlinear output regulation with adaptive internal model," IEEE Trans. Autom. Control, vol. 46, no. 8, pp. 1178-1194, 2001.

[15] F. D. Priscoli, L. Marconi, and A. Isidori, "A new approach to adaptive nonlinear regulation," SIAM J. Control Optim., vol. 45, no. 3, pp. 829-855, 2006.

[16] A. Isidori, L. Marconi, and L. Praly, "Robust design of nonlinear internal models without adaptation," Automatica, vol. 48, pp. 2409-2419, 2012.

[17] F. Forte, L. Marconi, and A. Isidori, "Robust design of internal models by nonlinear regression," in IFAC 9th Symposium on Nonlinear Control Systems, (Toulouse, France), pp. 283-288, 2013.

[18] M. Bin, D. Astolfi, and L. Marconi, "Robust internal model design by nonlinear regression via low-power highgain observers," in 2016 IEEE 56th Conf. on Decision and Control (CDC), (Las Vegas, USA), pp. 4740-4745, 2016.

[19] F. Forte, L. Marconi, and A. R. Teel, "Robust nonlinear regulation: Continuous-time internal models and hybrid identifiers," IEEE Trans. autom. control, vol. 62, no. 7, pp. 3136-3151, 2017.

[20] M. Bin and L. Marconi, "Adaptive output regulation via postprocessing internal models and hybrid identifiers," in 2017 IEEE 56th Conf. Decision and Control (CDC), (Melbourne, Australia), pp. 1478-1483, 2017.

[21] M. Bin and L. Marconi, "“class-type" identificationbased internal models in multivariable nonlinear output regulation," 2018. Under review. Preprint: arXiv:1805.05629.

[22] A. A. Feldbaum, "Dual control theory," Autom. Remote Control, vol. 21, no. 9, pp. 874-880, 1960.

[23] M. Gevers, "Identification for control: From the early achievements to the revival of experiment design," European Journal of Control, vol. 11, pp. 1-18, 2005.

[24] R. Goebel, R. G. Sanfelice, and A. R. Teel, Hybrid Dynamical Systems. Princeton, N. J.: Princeton University Press, 2012.
[25] C. Cai and A. Teel, "Characterization of input-to-state stability for hybrid systems," Systems 8 Control Letters, vol. 58, pp. 47-53, 2009.

[26] A. Isidori, Lectures in Feedback Design for Multivariable Systems. Springer International Publishing, 2017.

[27] J. P. Hespanha and A. S. Morse, "Stability of switched systems with average dwell-time," in 1999 IEEE 38th Conf. on Decision and Control, pp. 2655-2660, 1999.

[28] C. Cai, A. Teel, and R. Goebel, "Smooth Lyapunov functions for hybrid systems part II: (Pre)asymptotic stable compact sets," IEEE Trans. autom. control, vol. 53, pp. 734-748, 2008.

[29] D. Liberzon, D. Nešić, and A. R. Teel, "Lyapunov-based small-gain theorems for hybrid systems," IEEE Trans. autom. control, vol. 59, pp. 1395-1410, June 2014.

[30] C. Cai and A. Teel, "Robust input-to-state stability for hybrid systems," SIAM J. Control Optim., vol. 51, pp. 16511678,2013

[31] F. Deutsch, Best Approximation in Inner Product Spaces. Springer-Verlag, 2001.

[32] S. L. Campbell and C. D. Meyer, Generalized Inverses of Linear Transformations. SIAM, 2009. 